OPEN ACCESS

Edited by:

David Proud,

University of Calgary, Canada

Reviewed by:

Michael B. Fessler,

National Institute of Environmental Health Sciences (NIEHS),

United States

Alessandro Venosa,

The University of Utah, United States

${ }^{*}$ Correspondence:

Jeremy A. Hirota

hirotaja@mcmaster.ca

tThese two authors were equal in supervision of the staff throughout the entire study period

Specialty section:

This article was submitted to Microbes and Innate Immunity,

a section of the journal

Frontiers in Cellular and Infection

Microbiology

Received: 08 February 2020

Accepted: 05 August 2020

Published: 16 September 2020

Citation:

Cao QT, Aguiar JA, Tremblay BJ-M,

Abbas N, Tiessen N, Revill S,

Makhdami N, Ayoub A, Cox G, Ask K,

Doxey AC and Hirota JA (2020)

ABCF1 Regulates dsDNA-induced

Immune Responses in Human Airway Epithelial Cells.

Front. Cell. Infect. Microbiol. 10:487.

doi: 10.3389/fcimb.2020.00487

\section{ABCF1 Regulates dsDNA-induced Immune Responses in Human Airway Epithelial Cells}

\author{
Quynh T. Cao ${ }^{1}$, Jennifer A. Aguiar ${ }^{2}$, Benjamin J-M. Tremblay ${ }^{2}$, Nadin Abbas ${ }^{1}$, \\ Nicholas Tiessen ${ }^{1}$, Spencer Revill ${ }^{1}$, Nima Makhdami ${ }^{1}$, Anmar Ayoub ${ }^{1}$, Gerard Cox ${ }^{1}$, \\ Kjetil Ask ${ }^{1,3}$, Andrew C. Doxey ${ }^{1,2+}$ and Jeremy A. Hirota ${ }^{1,2,3,4 * \dagger}$
}

\begin{abstract}
${ }^{1}$ Division of Respirology, Department of Medicine, Firestone Institute for Respiratory Health, McMaster University, Hamilton, ON, Canada, ${ }^{2}$ Department of Biology, University of Waterloo, Waterloo, ON, Canada, ${ }^{3}$ McMaster Immunology Research Centre, McMaster University, Hamilton, ON, Canada, ${ }^{4}$ Division of Respiratory Medicine, Department of Medicine, University of British Columbia, Vancouver, BC, Canada
\end{abstract}

Background: The airway epithelium represents a critical component of the human lung that helps orchestrate defenses against respiratory tract viral infections, which are responsible for more than 2.5 million deaths/year globally. Innate immune activities of the airway epithelium rely on Toll-like receptors (TLRs), nucleotide binding and leucine-rich-repeat pyrin domain containing (NLRP) receptors, and cytosolic nucleic acid sensors. ATP Binding Cassette (ABC) transporters are ubiquitous across all three domains of life-Archaea, Bacteria, and Eukarya-and expressed in the human airway epithelium. ABCF1, a unique ABC family member that lacks a transmembrane domain, has been defined as a cytosolic nucleic acid sensor that regulates CXCL10, interferon- $\beta$ expression, and downstream type I interferon responses. We tested the hypothesis that ABCF1 functions as a dsDNA nucleic acid sensor in human airway epithelial cells important in regulating antiviral responses.

Methods: Expression and localization experiments were performed using in situ hybridization and immunohistochemistry in human lung tissue, while confirmatory transcript and protein expression was performed in human airway epithelial cells. Functional experiments were performed with siRNA methods in a human airway epithelial cell line. Complementary transcriptomic analyses were performed to explore the contributions of ABCF1 to gene expression patterns.

Results: Using archived human lung and human airway epithelial cells, we confirm expression of ABCF1 gene and protein expression in these tissue samples, with a role for mediating CXCL10 production in response to dsDNA viral mimic challenge. Although, ABCF1 knockdown was associated with an attenuation of select genes involved in the antiviral responses, Gene Ontology analyses revealed a greater interaction of ABCF1 with TLR signaling suggesting a multifactorial role for ABCF1 in innate immunity in human airway epithelial cells. 
Conclusion: ABCF1 is a candidate cytosolic nucleic acid sensor and modulator of TLR signaling that is expressed at gene and protein levels in human airway epithelial cells. The precise level where ABCF1 protein functions to modulate immune responses to pathogens remains to be determined but is anticipated to involve IRF-3 and CXCL10 production.

Keywords: ABCF1, airway epithelial cells, virus, CXCL10, innate immunity

\section{INTRODUCTION}

The human lung functions at the interface of the external and internal environments and is exposed to over 10,000 liters of air each day from normal respiration. The airway epithelium represents a critical component of the human lung that helps orchestrate defenses against inhaled noxious substances that may include air pollution, allergens, bacteria, and viral insults (Parker and Prince, 2011; Proud and Leigh, 2012; Hirota and Knight, 2013). To manage these continuous insults, the airway epithelium has evolved to be a multi-functional barrier tissue with mechanical and immunological impedances, manifested through the mucociliary ladder, protein-protein junctions, and innate immune processes. A dominant exposure important in both healthy individuals and those with underlying chronic respiratory diseases are viral infections. Collectively, respiratory tract viral infections are responsible for more than 2.5 million deaths/year globally and represent an economic burden on health care systems for all demographics (GBD 2017 Causes of Death Collaborators, 2018). In individuals with underlying chronic airway disease, respiratory tract viral infections increase frequency and severity of disease exacerbations, hospitalizations, and contribute to morbidity and mortality (Ritchie et al., 2015; Jartti and Gern, 2017; Singanayagam et al., 2018; Viniol and Vogelmeier, 2018; Linden et al., 2019). Understanding the mechanisms governing respiratory tract viral infections and host defense is essential for the future development of treatments aimed at minimizing the morbidity and mortality of these pathogens.

Innate immune activities of the airway epithelium rely on accurate sensing of the external environment. The threat posed by viruses that infect the respiratory mucosa is countered by the airway epithelium expressing functional Toll-like receptors (TLRs), nucleotide binding and leucine-rich-repeat pyrin domain containing (NLRP) receptors, and cytosolic nucleic acid sensors that are able to rapidly detect exposures and provide host defense (Parker and Prince, 2011; Hirota et al., 2012; Proud and Leigh, 2012; Hirota and Knight, 2013; Bailey et al., 2015; Zhu et al., 2019). Antiviral sensing mechanisms in the respiratory mucosa enable responses to influenza A, respiratory syncytial virus, rhinovirus, and human parainfluenza virus; all single stranded RNA viruses (Hodinka, 2016). dsDNA viruses are also relevant lung infections, with adenovirus capable of inducing influenza like symptoms in healthy subjects and associated with chronic respiratory disease exacerbations (Kokturk et al., 2015; Biggs et al., 2018; Carlin, 2019; Linden et al., 2019). Like RNA viruses, adenovirus is able to infect airway epithelium followed by replication, which leads to a variety of innate immune defenses able to sense viral nucleic acids and proteins (Bergelson et al., 1997; Bewley et al., 1999; Carlin, 2019). Vaccinia virus is another dsDNA virus that is able to infect airway epithelium and has been explored for capacity to genetically engineer the virus for transgene delivery, vaccination strategies, and studying Variola virus infections (Rich et al., 1990; Vermeer et al., 2007; Le Boeuf et al., 2010; Altenburg et al., 2018; de Vries et al., 2018). Exploring how the airway epithelium responds to viruses may provide new strategies for controlling infections, optimizing transgene delivery, and vaccination strategies relevant in lung health and disease.

ATP Binding Cassette $(\mathrm{ABC})$ transporters are ubiquitous across all three domains of life-Archaea, Bacteria, and Eukarya (Vasiliou et al., 2009). In humans, the $49 \mathrm{ABC}$ transporters are classified according to structure and function, resulting in seven families. $A B C$ transporters with clear involvement in lung health and disease include $A B C A 3$ and ABCC7 (better known as cystic fibrosis transmembrane conductance regulatorCFTR), responsible for surfactant production and ion transport, respectively (Riordan et al., 1989; Rich et al., 1990; Shulenin et al., 2004). The $\mathrm{ABCF}$ family members are unique in their structure and function as they lack transmembrane regions and therefore lack capacity for transport of substrates (Richard et al., 1998; Vasiliou et al., 2009). Of the ABCF family members, ABCF1 is most extensively characterized in eukaryotes, with functions ranging from initiation of mRNA translation, immune modulation, and nucleic acid sensing (Richard et al., 1998; Tyzack et al., 2000; Paytubi et al., 2008, 2009; Lee et al., 2013; Arora et al., 2019). The diverse functions attributed to $\mathrm{ABCF} 1$ are physiologically important, as demonstrated by the embryonic lethality of homozygous deletion of ABCF1 in mice (Wilcox et al., 2017). To date, nucleic acid sensing by ABCF1 has been defined using the dsDNA immunostimulatory DNA (ISD) sequence derived from Listeria monocytogenes (Stetson and Medzhitov, 2006) and a dsDNA HIV sequence, with both nucleic acid motifs inducing CXCL10, interferon$\beta$ expression, and downstream type I interferon responses in mouse embryonic fibroblasts (Lee et al., 2013). Complementary to dsDNA sensing, immune modulation mediated by ubiquitinconjugating activities of $\mathrm{ABCF} 1$ have been defined in the context of macrophage polarization and immune responses linked to interferon- $\beta$ production and tolerance important in mouse models of sepsis (Arora et al., 2019). In the context of studies using human lung samples, $A B C F 1$ gene expression has been identified in the human airway epithelium (Aguiar et al., 2019), although confirmation of protein and function remains to be 
determined. The clear in vivo demonstration of ABCF1 functions in immune responses in mouse models and the presence of detectable $A B C F 1$ gene expression the human airways warrants a deeper interrogation into the expression and function of this molecule in human health and disease.

Defining defense mechanisms in airway epithelial cells has important consequences in both lung health and disease, with the potential for interventions that could reduce viral-induced pathologies and exacerbations of chronic respiratory diseases (Ritchie et al., 2015; Jartti and Gern, 2017; Singanayagam et al., 2018; Viniol and Vogelmeier, 2018; Linden et al., 2019). We therefore tested the hypothesis that ABCF1 functions as a dsDNA nucleic acid sensor in human airway epithelial cells important in regulating antiviral responses, using archived human lung samples and human airway epithelial cells. Expression and localization experiments were performed using in situ hybridization and immunohistochemistry in human lung tissue, while confirmatory transcript and protein expression was performed in human airway epithelial cells. Functional experiments were performed with siRNA methods as no selective small molecule inhibitors to ABCF1 have been validated to date. Complementary transcriptomic analyses were performed to explore the potential contributions of ABCF1 beyond dsDNA virus sensing. A focused approach on CXCL10 as a readout was performed based on the discovery of ABCF1 as a dsDNA sensor in mouse embryonic fibroblasts. Further hypothesis-free analyses explored candidate pathways differentially regulated during $\mathrm{ABCF} 1$ attenuation. Our results confirm expression of ABCF1 in human airway epithelial cells with a role for mediating CXCL10 production in response to dsDNA viral mimic challenge. Although, reduced expression of ABCF1 was associated with an attenuation of select genes involved in the antiviral responses, Gene Ontology analyses revealed a greater interaction of $\mathrm{ABCF} 1$ with TLR signaling suggesting a multifactorial role for $\mathrm{ABCF} 1$ in innate immunity in human airway epithelial cells.

\section{METHODS}

\section{Human Ethics}

All studies using primary human lung material were approved by Hamilton integrated Research Ethics Board (HiREB-5305-T and 5099-T).

\section{Reagents}

In situ hybridization was performed using a custom RNAscope $^{\mathrm{TM}}$ probe for ABCF1 (construct targeting 17132726 of NM_001025091.1) generated by Advanced Cell Diagnostics (ACD, Newark, California). Negative and positive control probes for quality control of RNA signal in analyzed human tissues were purchased from ACD (data not shown). Protein cell lysates were collected by lysing and scraping cells with RIPA Lysis buffer (VWR, Mississauga, Ontario) mixed with protease inhibitor cocktail (Sigma-Aldrich, Oakville, Ontario). Immunoblots were conducted using Mini-Protean TGX stain-free gels and Transfer-Blot Turbo RTA Transfer Kit reagents (Bio-Rad, Mississauga, Ontario). ABCF1 protein was probed with primary anti-ABCF1 antibody (HPA017578, Sigma-Aldrich, Oakville, Ontario) at 1:100 in 3\% Casein in $1 X$ Tris Buffered Saline with TWEEN () 20 (Sigma-Aldrich, Oakville, Ontario, and Anti-rabbit HRP-linked Antibody (7074S-Cell Signaling Technology, Danvers, MA) at 1:2000. Immunohistochemistry was performed using the same antiABCF1 antibody as immunoblotting. ABCF1 and scramble siRNA SMARTpool siGENOME transfection reagents were purchased from Dharmacon (M-008263-01 and D-001206-14, Lafayette, Colorado). The SMARTpool siRNA reagent is a pool of 4 siRNA duplexes all designed to target distinct sites within the specific gene of interest. The 4 different siRNA within the pool were selected by Dharmacon using their design algorithm to have the optimal silencing of the target transcript NM_001025091 and NM_001090, with all siRNA targeting within the open reading frame. Cell viability was estimated with the Pierce LDH Cytotoxicity Assay kit (ThermoFisher Scientific, Mississauga, Ontario). RNA samples were lysed with Buffer RLT and purified with RNeasy Mini Kit columns (Qiagen, Toronto, Ontario). The ligands ISD, ISD control, VACV-70, VACV-70 control, and Poly:IC were purchased from Invivogen (San Diego, California). Apart from Poly:IC, where it was applied directly to the cells, the other ligands were complexed with LyoVec transfection reagent (Invivogen, San Diego, California). Human CXCL10 was quantified using a commercial ELISA with ancillary reagent kit (R\&D Systems, Oakville, Ontario). The protocol for quantifying CXCL10 was modified with the use of a loading plate for the samples.

\section{Cell Culture}

All experiments were performed in submerged monolayer cell culture. An immortalized human airway epithelial cell line (HBEC-6KT) over expressing human telomerase reverse transcriptase (hTERT) and cyclin-dependent kinase 4 (Cdk4) was used as previously described (Ramirez et al., 2004; Hirota et al., 2015a,b; Huff et al., 2017a,b). The cell line was obtained from lung biopsies that were not histologically involved with lung cancer from non-smoker donors and it does not have a malignant phenotype (Ramirez et al., 2004). HBEC-6KT were grown in keratinocyte serum free medium (ThermoFisher Scientific, Mississauga, Ontario) supplemented with $0.8 \mathrm{ng} / \mathrm{ml}$ epithelial growth factor, $50 \mu \mathrm{g} / \mathrm{ml}$ bovine pituitary extract and $1 \times$ penicillin/streptomycin (Sigma-Aldrich, Oakville, Ontario). Calu-3 cells (ATCC HTB-55) were grown in Eagle's Minimum Essential Media supplemented with $10 \%$ fetal bovine serum (Wisent, Saint-Jean-Baptiste, QC), $1 \mathrm{mM} \mathrm{HEPES,} \mathrm{and} 1 \times$ penicillin/streptomycin. Primary human bronchial epithelial cells derived from healthy patient bronchial brushings were grown in PneumaCult ExPlus Medium supplemented with $96 \mu \mathrm{g} / \mathrm{ml}$ hydrocortisone (StemCell Technologies, Vancouver, $\mathrm{BC}$ ) and $1 \times$ antimicrobial-antimycotics (ThermoFisher Scientific, Mississauga, Ontario). All cells were grown at $37^{\circ} \mathrm{C}$ at $5 \% \quad \mathrm{CO}_{2}$. Experiments with primary cells were performed between passages 1 and 4 , and experiments with HBEC-6KT and Calu-3 cells were performed within 5 passages. 


\section{In vitro Experiments}

All in vitro knockdown experiments in HBEC-6KT were done using siRNA transfected with DharmaFECT Transfection Reagent according to the manufacturer's instructions. Cells were transfected with siABCF1 or siCTRL for $24 \mathrm{~h}$. For non-challenge experiments, cells were immediately collected for outcome measurements. For challenge experiments, after knockdown, cells were transfected with an immunostimulatory ligand for $24 \mathrm{~h}$ followed by outcome measurements of cell viability (LDH and cell morphology), function (cytokine secretion), protein expression (immunoblot), or gene transcription (microarray). For TNF- $\alpha$ stimulation experiments, $10 \mathrm{ng} / \mathrm{ml}$ was incubated for $24 \mathrm{~h}$ followed by protein collection for immunoblots. For ISD and VACV-70 stimulation experiments, a concentration-response study was performed using $0.316-3.16 \mu \mathrm{g} / \mathrm{ml}$ (ISD) or $0.1-$ $3.16 \mu \mathrm{g} / \mathrm{ml}$ (VACV-70) followed by incubation for $24 \mathrm{~h}$. For Poly I:C stimulation experiments, $1 \mu \mathrm{g} / \mathrm{ml}$ was incubated for $24 \mathrm{~h}$.

\section{Cytokines Analysis}

Cell supernatants were collected following in vitro experiments and sent to Eve Technologies for a Human Cytokine Array/Chemokine Array 48-Plex (Eve Technologies, Calgary, Alberta). Eve Technologies uses the Bio-Plex ${ }^{\circledR} 200$ to detect 49 different cytokines, chemokines and growth factors: sCD40L, EGF, Eotaxin, FGF-2, Flt-3 ligand, Fractalkine, G-CSF, GM-CSF, GRO $\alpha$, IFN $\alpha 2$, IFN $\gamma$, IL-1 $\alpha$, IL-1 $\beta$, IL-1ra, IL-2, IL-3, IL-4, IL-5, IL-6, IL-7, IL-8, IL-9, IL-10, IL-12 (p40), IL-12 (p70), IL-13, IL-15, IL-17A, IL-17E/IL-25, IL-17F, IL-18, IL-22, IL-27, IP-10 (CXCL10), MCP-1, MCP-3, M-CSF, MDC (CCL22), MIG, MIP- $1 \alpha$, MIP- $1 \beta$, PDGF-AA, PDGF-AB/BB, RANTES (CCL5), TGF- $\alpha$, TNF- $\alpha$, TNF- $\beta$, VEGF-A.

\section{Histology, Digital Slide Scanning, and Microscopy}

In situ hybridization and immunohistochemistry was performed using a Leica Bond $\mathrm{Rx}$ autostainer with instrument and application specific reagent kits (Richmond Hill, Ontario). The human lung tissues selected for analysis were formalin fixed and paraffin embedded. Lung samples from archived hospital clinical samples were determined to be free of lung pathology and were collected during a tumor resection procedure. No history on smoking was available for the samples analyzed. Following selection, four micron thick sections were stained with using RNAscope ${ }^{\mathrm{TM}}$ probes (in situ hybridization) or antibody (immunohistochemistry) following directions supplied with the Leica Bond reagent kits. For IHC, heat-induced antigen retrieval in citrate buffer was performed at $\mathrm{pH} 6$ with primary antibody diluted at 1:50. Slides underwent digital slide scanning using an Olympus VS120-L100 Virtual Slide System at $40 \times$ magnification with VS-ASW-L100 V2.9 software and a VC50 color camera (Richmond Hill, Ontario). Image acquisition and formatting was performed using Halo Software (Indica Labs, Albuquerque, NM).

\section{Gene Expression Omnibus (GEO) Dataset Mining}

Gene expression patterns of $A B C F 1$ in human airway epithelial cells was determined relative to markers for immune cells
(CD34), ABC transporters of known function in airway epithelial cells $(A B C C 4, A B C C 7)$, and junctions $(C D H 1)$ in a dataset containing samples from trachea, large airways (generation $2^{\text {nd }}$ $3^{\text {rd }}$ ), and small airways (generation $10^{\text {th }}-12^{\text {th }}$ ) from healthy subjects (GSE11906, Affymetrix Human Genome U133 Plus 2 microarray platform) (Raman et al., 2009). The following probesets were used to extract gene expression data: $A B C F 1$ (200045_at), ABCC4 (203196_at), ABCC7 (CFTR; 205043_at), CDH1 (201131_s_at), and CD34 (209543_s_at). In cases where more than one probe corresponded to a given gene, the following hierarchy was used to select an individual probe for further use: perfect, unique matches (probes ending in _at or_a_at) were preferred over mismatch or non-unique probes (ending in_s_at or_x_at). GSE11906 included 17 trachea (age-42 +/- 7), 21 large airway (age $-42+/-9$ ), and 35 small airway samples.

\section{Processing of Raw Microarray Data}

Raw intensity values from a microarray experiment using the Affymetrix Clariom S Human chip-type were imported into the $\mathrm{R}$ statistical language environment (version 3.6.1; R Core Team, 2019). Probe definition files were obtained from the Brainarray database [version 24 Dai et al. (2005)]. The Single Channel Array Normalization (SCAN) method was used to obtain $\log _{2}$-transformed normalized expression values with the SCAN.UPC R package [version 2.26.0 Piccolo et al. (2012)], with annotation data from the Bioconductor project [version 3.9 Huber et al. (2015)]. The microarray data generated from ABCF1 siRNA experiments is deposited in GEO with accession number GSE150541.

\section{Analysis of Processed Microarray Data}

From the processed expression values, principle component analyses were performed with the prcomp function (version 0.1.0) from the R statistical language (version 3.6.1; R Core Team, 2019) using default parameters. Determination of statistically significant differential gene expression was performed using the empirical Bayes method via the eBayes function from the limma R package [version 3.40.0 (Ritchie, M. E. et al., 2015)]. $P$-values were adjusted using the Benjamini \& Hochberg method, with a significance cutoff of 0.05 . Significantly enriched Gene Ontology (GO) Biological Process Terms (ranked by p-value) were determined using Enrichr (Chen et al., 2013; Kuleshov et al., 2016). Scatter plots, PCA plots, and GO term enrichment dot plots were generated using the ggplot2 $\mathrm{R}$ package (version 3.2.1). Heat maps were generated using the pheatmap $\mathrm{R}$ package (version 1.0.12), with $\log _{2}$ expression scaled by gene and complete hierarchical clustering using a Euclidean distance measure applied. A GO term enrichment clustergram was modified from Enrichr using Inkscape.

\section{Statistical Analyses}

All experiments were performed with an $n \geq 3$ unless otherwise noted. Experiments with HBEC-6KT and Calu-3 cells were considered independent when separated by a passage. Statistics were determined by permutation ANOVA with a Bonferonnicorrected post-hoc test comparing selected groups with $p<0.05$ determined to be statistically significant. 


\section{RESULTS}

\section{ABCF1 Gene and Protein Expression Is Localized to Human Airway Epithelial Cells in situ and in vitro}

Expression and functional studies of ABCF1 have focused on human synoviocytes, mouse embryonic fibroblasts, human embryonic kidney cells, and peripheral blood mononuclear cells (Richard et al., 1998; Tyzack et al., 2000; Paytubi et al., 2008, 2009; Arora et al., 2019). We have demonstrated gene expression of ABCF1 in human airway epithelial cells (Aguiar et al., 2019). To date, no in situ gene and protein expression data has confirmed $\mathrm{ABCF} 1$ expression in human lung tissues.

To address this knowledge gap, we first mined publicly available gene expression data from primary human airway epithelial cells from healthy subjects. ABCF1 gene expression was observed along the airway generations (trachea, large, and small) at levels relative to $A B C C 7 / C F T R$ and $A B C C 4$, two other $\mathrm{ABC}$ transporters with reported functions in airway epithelial cells (Riordan et al., 1989; Conner et al., 2013; Gold et al., 2016; Huff et al., 2017b) (Figure 1A). CD34 and CDH1 (encoding E-Cadherin) were used as negative and positive control genes, respectively, for airway epithelial cells to provide contextual expression levels.

Next, in situ localization of ABCF1 gene transcript was performed using RNAscope ${ }^{\mathrm{TM}}$ probes on archived formalin fixed paraffin embedded human lung samples (Figure 1B). ABCF1 gene transcript was observed in small puncta throughout the cytoplasm and nuclear areas of airway epithelial cells. ABCF1 staining was also observed in submucosal cells with morphology consistent with macrophages.

Protein expression levels were next explored with validation of a commercially available antibody for ABCF1. Positive staining was observed in human airway epithelial cells as shown in a serial section used for in situ hybridization (Figure 1C) with sparse staining in immune cells with macrophage morphology. Using in vitro culture of primary human airway epithelial cells and two distinct airway epithelial cell lines (Figure 1D). For each airway epithelial cell type, a single band was observed at the predicted molecular weight of $96 \mathrm{kDa}$ for $\mathrm{ABCF} 1$, validating the use of the antibody for in situ immunohistochemistry localization.

Lastly, to explore proposed regulatory mechanisms for ABCF1 (Richard et al., 1998), we performed a TNF- $\alpha$ exposure in human airway epithelial cells. Exposure to $10 \mathrm{ng} / \mathrm{ml} \mathrm{TNF}-\alpha$ for $24 \mathrm{~h}$ failed to induce a change in $A B C F 1$ protein expression (Figures 1E,F), despite inducing an increase in IL-8 (Figure 1G).

Collectively our in vitro and in situ data confirm gene and protein expression of ABCF1 in human airway epithelial cells, warranting downstream characterization and functional studies.

\section{Reduced Expression of ABCF1 Under Basal Conditions in vitro Has Limited Impact on HBEC-6KT Viability and Transcriptional Profiles}

Functional studies have implicated ABCF1 in translation initiation and have demonstrated that homozygous loss of function results in embryonic lethality (Tyzack et al., 2000; Paytubi et al., 2008, 2009; Wilcox et al., 2017). We therefore first interrogated the basal functions of ABCF1 in our human airway epithelial cells in the context of cell viability and transcriptional profiling.

We performed siRNA experiments to reduce ABCF1 expression levels as no small molecule ABCF1 inhibitor has been described to date. Using siRNA approaches in HBEC-6KT, we confirm that $\mathrm{ABCF} 1$ protein levels can be reduced with qualitative (Figure 2A) and quantitative measures (Figure 2B). LDH levels were not elevated when ABCF1 expression was reduced with siRNA Figure 2C. Cell morphology was not different in human airway epithelial cells with reduced ABCF1 expression (Figure 2D). Collectively, the quantitative and qualitative data suggest moderate levels of siRNA knockdown are not associated with compromised HBEC-6KT viability under basal conditions.

To interrogate the impact of reduced expression of $\mathrm{ABCF} 1$ under basal conditions, a human gene expression microarray analysis was performed. A principal component analysis of $A B C F 1$ reduction and corresponding experimental controls revealed no clustering between experimental replicates for any condition (Figure 2E), suggesting that the overall impact of $A B C F 1$ reduction under basal conditions minimally impacted global gene expression patterns. Statistical analysis comparing ABCF1 reduction and siRNA control treated HBEC-6KT confirmed $A B C F 1$ gene was down-regulated (Figure 2F) which was associated with only one other significantly differentially expressed (up or down) gene, C12orf75, which encodes overexpressed in colon carcinoma-1 (OCC-1) protein.

Collectively our in vitro studies under basal conditions demonstrate that reduced ABCF1 expression is not associated with changes in viability or significant genome wide changes in transcriptional profiles in HBEC-6KT.

\section{The dsDNA Viral Mimic VACV-70 Induces CXCL10 and an Antiviral Response in HBEC-6KT in vitro}

Since reduced expression of ABCF1 under basal conditions resulted in limited impacts on cell viability and gene transcription, we next explored conditions of challenge in HBEC-6KT. ABCF1 was described as a dsDNA sensor in mouse embryonic fibroblasts that mediated CXCL10 secretion under challenge conditions with the viral mimic interferon stimulatory DNA (ISD) sequence (Lee et al., 2013), a 45bp oligomer shown to activate the STING-TBK1-IRF3 antiviral sensing axis (Stetson and Medzhitov, 2006; Ishikawa et al., 2009).

To determine the response of HBEC-6KT to ISD, we performed a concentration-response study followed by quantification of extracellular CXCL10 secretion (Figure 3A). ISD induced an increase in CXCL10 at $1 \mu \mathrm{g} / \mathrm{ml}$ while no increases were observed at lower $(0.316 \mu \mathrm{g} / \mathrm{ml})$ and higher $(3.16$ $\mu \mathrm{g} / \mathrm{ml}$ ) concentrations. Importantly, as concentration of ISD increased, the cellular response to the control (ssDNA of the ISD sequence) also increased. These results limited the use of ISD 


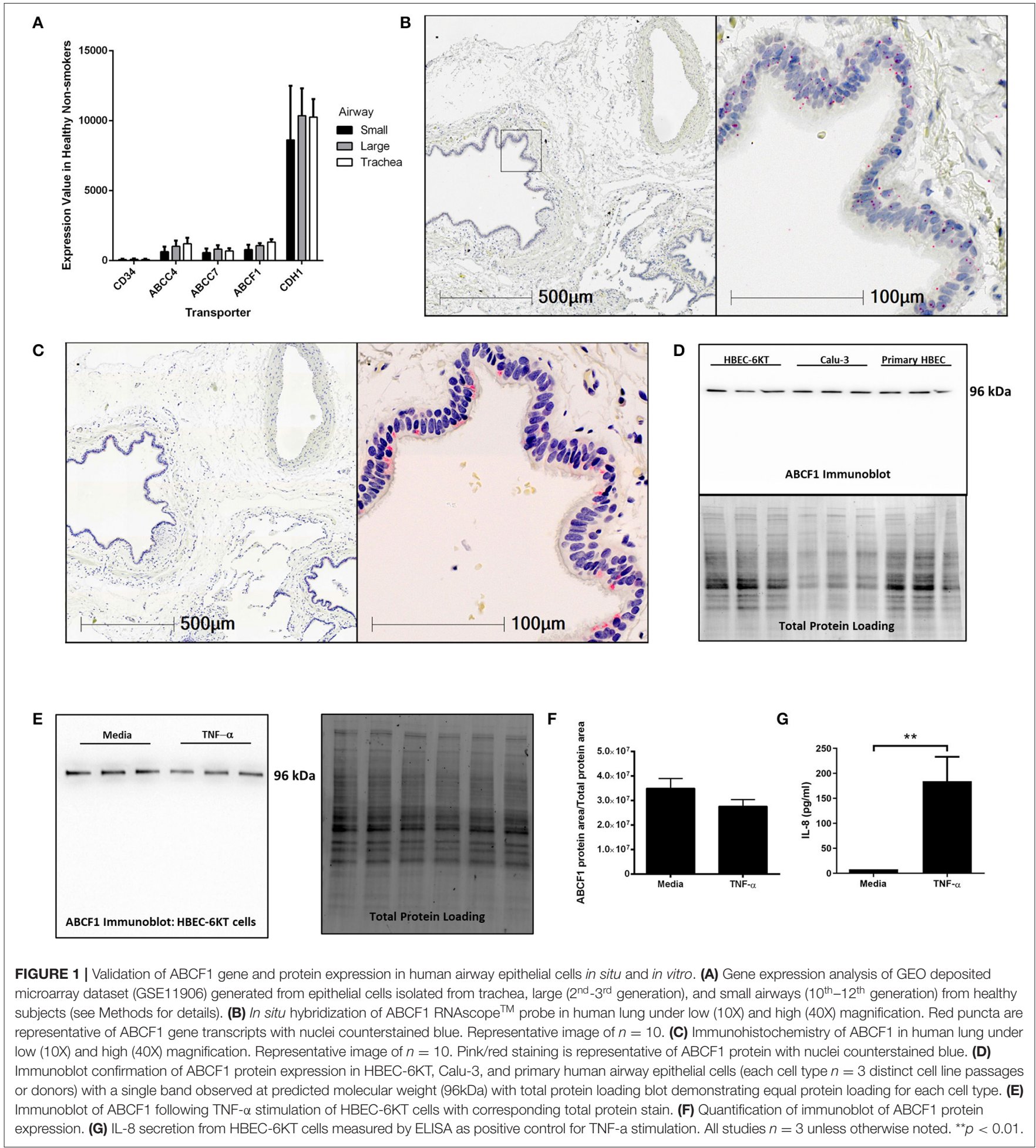

as dsDNA challenge stimulus in HBEC-6KT cells for studying ABCF1 function.

Vaccinia virus is a dsDNA virus that is able to infect airway epithelial cells (Rich et al., 1990; Vermeer et al., 2007; Le
Boeuf et al., 2010; Altenburg et al., 2018; de Vries et al., 2018). We therefore determined the response of HBEC-6KT to VACV-70, a 70bp dsDNA oligonucleotide containing Vaccinia virus motifs (Unterholzner et al., 2010). VACV-70 induced a 
A

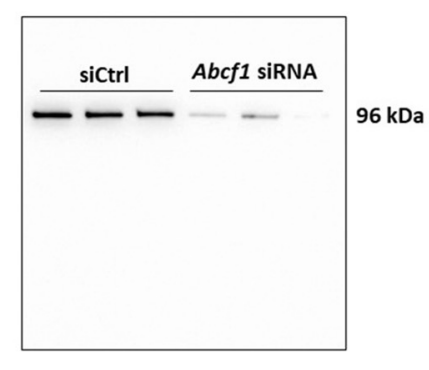

C

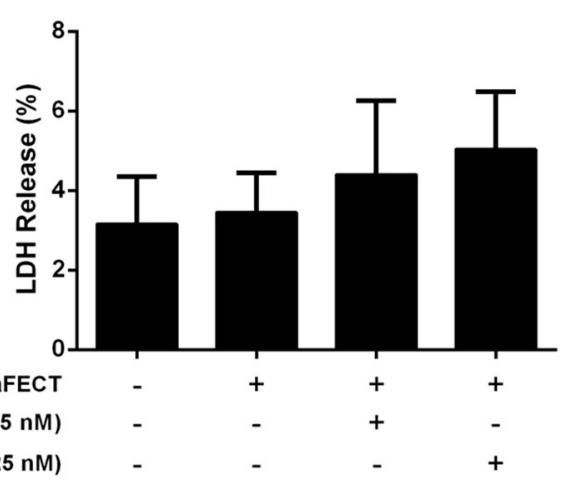

B

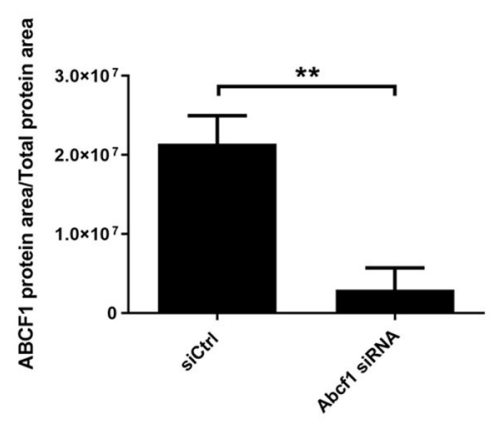

D

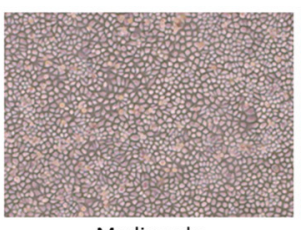

Media only

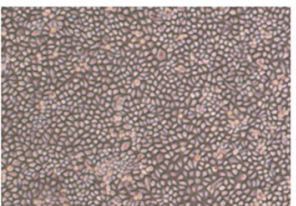

siCtrl/DharmaFECT

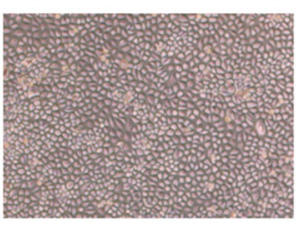

DharmaFECT
E

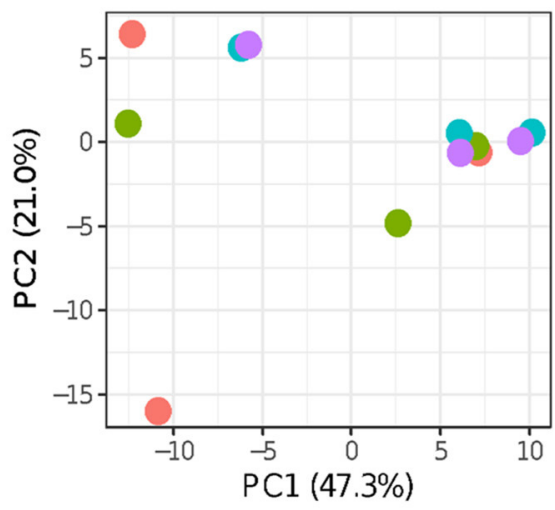

Media-only

- Transfection Reagent Only

Transfection Reagent and Control siRNA

Transfection Reagent and ABCF1 siRNA
$\mathbf{F}$

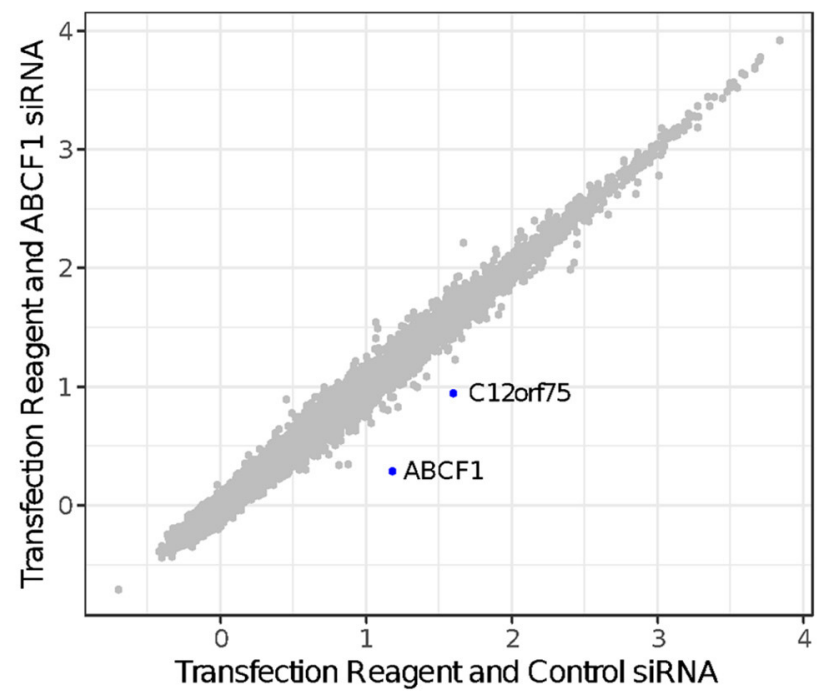

FIGURE 2 | Interrogation of ABCF1 under basal conditions in HBEC-6KT in vitro. (A) Immunoblot confirming siRNA-mediated knockdown of ABCF1 protein expression in HBEC-6KT cells. (B) Quantification of ABCF1 protein expression following siRNA treatment. (C) LDH quantification as a measure of cell viability following siRNA treatment. (D) Phase-contrast microscopy (4X magnification) of HBEC-6KT following siRNA treatment. (E) PCA plot of microarray gene expression profiles of HBEC-6KT cells following siRNA treatment. Red circles (media alone), green circles (transfection reagent only), blue circles (transfection reagent and control siRNA), purple circles (transfection reagent and ABCF1 siRNA). (F) Log2 expression data for transfection reagent with ABCF1 siRNA compared to transfection reagent with control siRNA. Significantly differently expressed genes are in blue and are down-regulated (ABCF1 and C12orf75). All studies $n=3 .{ }^{* \star} p<0.01$.

concentration dependent increase in CXCL10 from $0.316 \mu \mathrm{g} / \mathrm{ml}$ to $3.16 \mu \mathrm{g} / \mathrm{ml}$. In contrast to ISD, no cellular response to the control (ssDNA of the VACV-70 sequence) was observed at any concentration.
To characterize the molecular pathways activated by VACV-70, we performed a transcriptional and pathway analysis of HBEC-6KT following challenge. To interrogate the VACV-70 transcriptional responses a principal component 


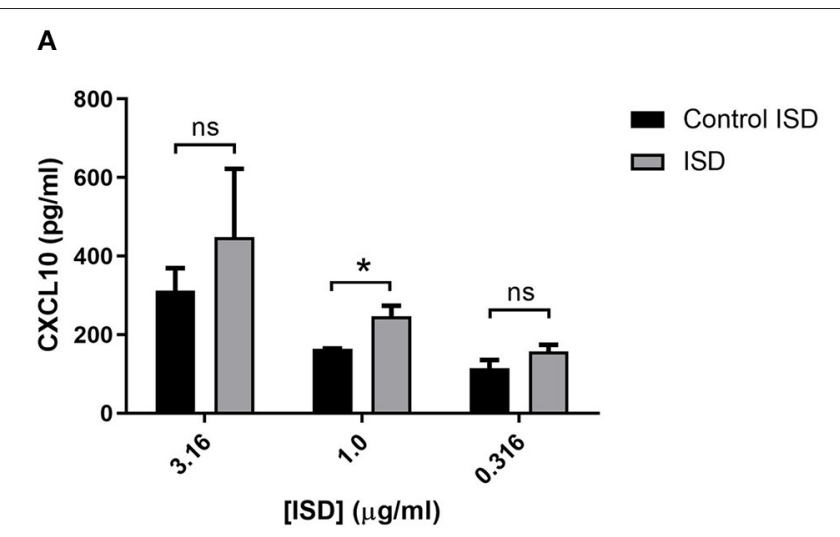

B

C
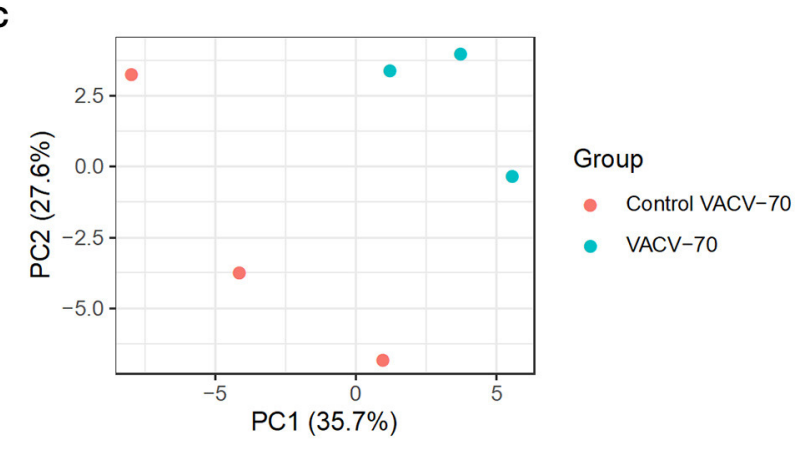

E

$\mathbf{F}$
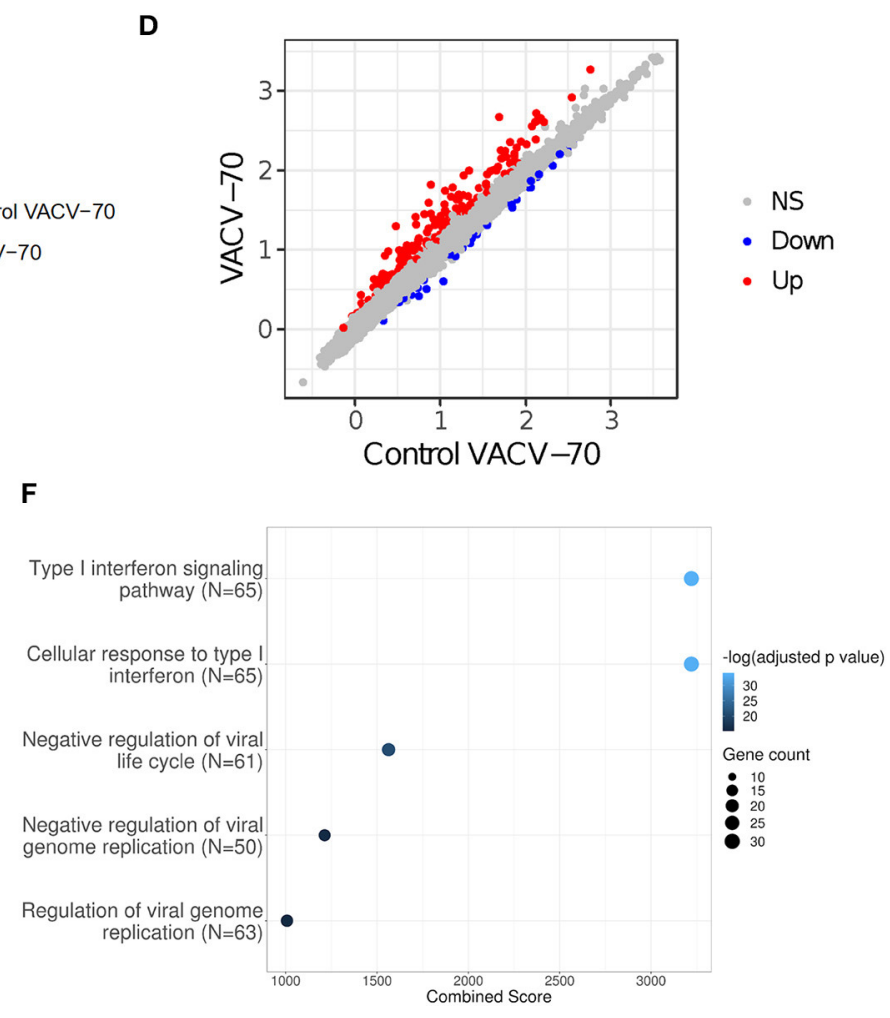

FIGURE 3 | dsDNA induced antiviral responses in HBEC-6KT in vitro. (A) Concentration-response analysis of ISD-induced CXCL10 protein production for HBEC-6KT cells. Gray bars: ISD, Black bars: control SsDNA generated from ISD. (B) Concentration-response analysis of VACV-70-induced CXCL10 protein production for HBEC-6KT cells. Gray bars: VACV-70, Black bars: control ssDNA generated from VACV-70. (C) PCA plot of microarray gene expression profiles of HBEC-6KT cells following transfection with VACV-70 or control. Red circles (control VACV-70), blue circles (VACV-70). (D) Log 2 expression data for transfection treatment with VACV-70 compared to control VACV-70. Significantly differently expressed genes are identified in blue (down-42 genes) and red (up-170 genes). (E) Heat map of $\log _{2}$ expression data (scaled by gene) of select known antiviral for VACV-70 and control VACV-70 samples. (F) Top 5 GO Biological Processes are ranked by increasing $-\log _{10}$ adjusted $p$-value, with number (Count) of significantly differentially expressed genes between VACV-70 and control VACV-70 contributing to the total number of genes associated with the given pathway $(M)$ denoted by the size of circle. All studies $n=3 ;{ }^{*} p<0.05$.

analysis was performed for microarray gene expression data, revealing distinct clustering between stimulation (VACV-70) and control (Figure 3C). Statistical analysis revealed 170 up-regulated genes and 42 down-regulated genes with VACV-70 stimulus (Figure 3D). VACV-70 upregulated CXCL10 gene expression and a curated list of antiviral related interferon stimulated genes (Figure 3E).
GO term analysis revealed that the top pathways activated by VACV-70 were associated with type I interferon signaling, viral responses, and cellular responses to viruses (Figure 3F).

Collectively our in vitro challenge studies confirm that VACV70, a dsDNA viral mimic, can induce CXCL10 and antiviral transcriptional responses in HBEC-6KT. 


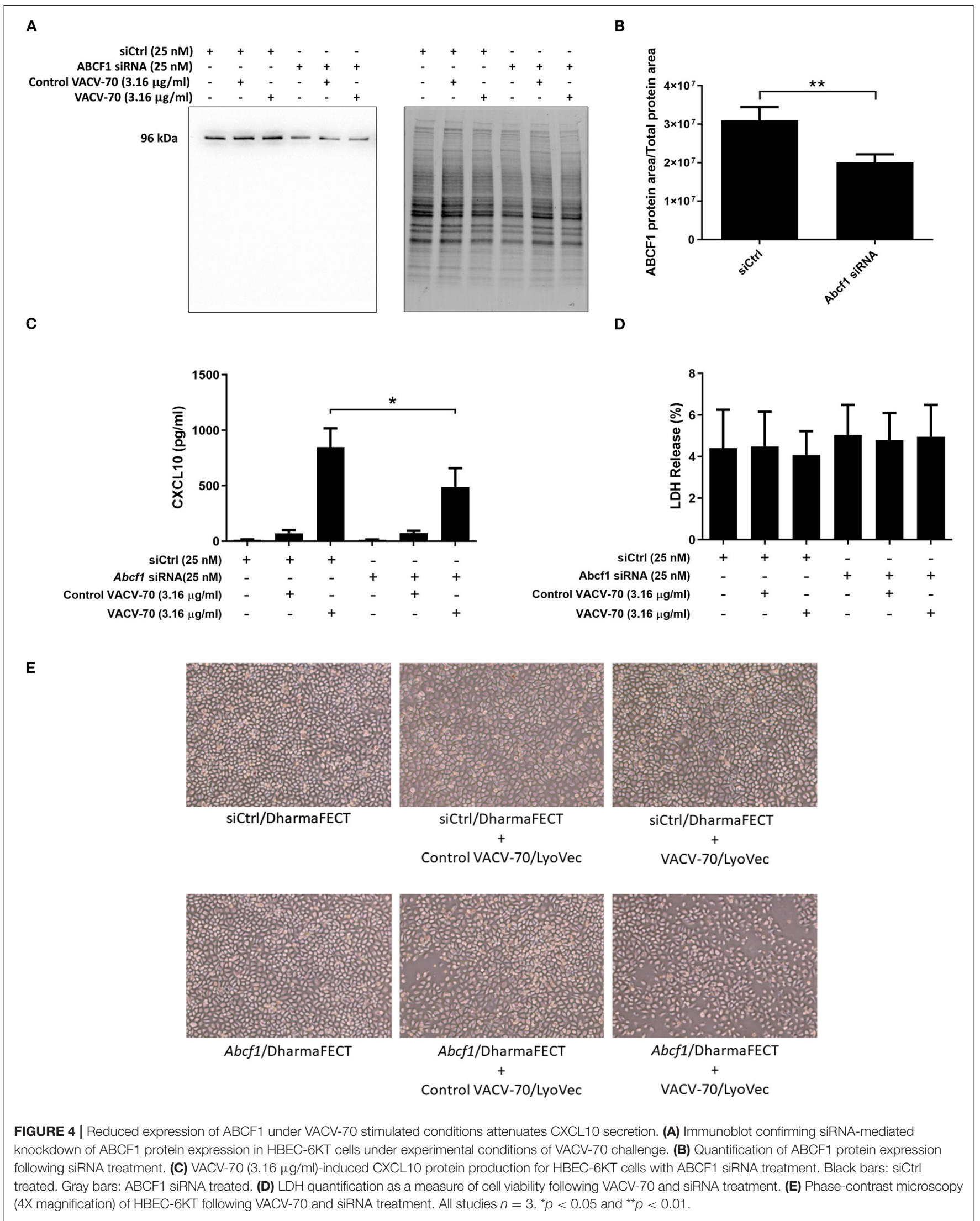




\section{Reduced Expression of ABCF1 Under VACV-70 Stimulated Conditions Attenuates CXCL10 Secretion With Limited Impact on HBEC-6KT Viability}

We have confirmed VACV-70 induction of CXCL10 in HBEC-6KT at the gene Figure 3E and protein (Figure 3B) levels. Furthermore, we have demonstrated siRNA-mediated reduction of $A B C F 1$ expression with no impact on cell viability (Figures 2A-D). We therefore performed a VACV-70 challenge with reduced expression of ABCF1 by siRNA with a primary readout of CXCL10.

ABCF1 reduction was associated with a decrease in CXCL10 protein secretion from HBEC-6KT, with confirmation and quantification of $\mathrm{ABCF} 1$ reduction performed by immunoblot (Figures 4A-C). Cell viability following VACV-70 challenge and ABCF1 attenuation was not impacted as assessed by LDH quantification (Figure 4D). Qualitative analysis following VACV70 challenge and ABCF1 revealed no impact on HBEC-6KT cell morphology (Figure 4E).

Collectively our in vitro challenge and functional studies demonstrate that ABCF1 siRNA treatment attenuated VACV-70induced CXCL10 protein secretion in HBEC-6KT.

\section{ABCF1 Reduction Does Not Impact VACV-70-Induced Antiviral Transcriptional Responses in HBEC-6KT}

In parallel to induction of CXCL10 gene, we have confirmed with GO pathway analysis that VACV-70 induces a dominant antiviral transcriptional signature (Figures 3E,F). We therefore next explored how reduced expression of $A B C F 1$ impacts transcriptional responses induced by VACV-70, beyond induction of CXCL10.

A principal component analysis was performed for microarray gene expression data, revealing distinct clustering in samples where ABCF1 expression was reduced relative to control conditions under conditions of VACV-70 challenge (Figure 6A green vs. purple). Statistical analysis revealed 63 up-regulated genes and 65 down-regulated genes when comparing $\mathrm{ABCF} 1$ reduction relative to control under conditions of VACV-70 challenge (Figure 6B). siRNA mediated reduction of ABCF1 was confirmed and associated with attenuation of CXCL10 gene expression (Figure 6C, $p=0.06$ ).

To explore a focused transcriptional response of ABCF1 reduction in the context of VACV-70 challenge, a hypothesisdirected approach curated 79 genes from the GO term "Regulation of defense response to virus" and key components of viral sensing for heat map visualization (Goubau et al., 2013) (Figure 6D). Statistical analysis revealed no global significant difference between $\mathrm{ABCF} 1$ reduction and control groups for the expression pattern of this curated list of genes.

To explore the broader transcriptional responses of ABCF1 reduction in the context of VACV-70 challenge, a hypothesisfree directed approach with GO term analysis was performed. Top-ranking GO pathway terms included Regulation of toll-like receptor 3-4 signaling pathways, which were driven by the genes WDFY1, TNFAIP3, and NR1D1 (Figures 6E,F). Complementary cytokine analysis further revealed impacts of $A B C F 1$ reduction on PDGF-BB, VEGF-A, and to a lesser extent IL-6, IL-8, and IL-1 family members, IL-1a, IL-18, and IL-1RA (Figure 5).

As our data suggested that ABCF1 functions in HBEC-6KT may extend beyond sensing of VACV-70 dsDNA viral mimic through regulation of TLR signaling, we explored Poly I:C, a dsRNA analog and TLR3 ligand that induces interferon responses including CXCL10 production. ABCF1 reduction was associated with a $63 \%$ reduction in Poly I:C-induced CXCL10 protein $(p=$ $0.07)$ secretion but did not impact Poly I:C-induced IL-8 protein secretion (Figures 6G,H).

Collectively our in vitro challenge and functional studies with transcriptional analyses demonstrate a role for $\mathrm{ABCF} 1$ in mediating VACV-70 and Poly I:C induced CXCL10 secretion and TLR3 related signaling in HBEC-6KT.

\section{DISCUSSION}

The human airway epithelium expresses a variety of sensors that can detect and initiate an immune response to virus infection. Recognition by these sensors can trigger downstream activation of antiviral responses by inducing the production and release of antiviral and inflammatory cytokines (Kawai and Akira, 2006). This includes TLRs found at the cell surface such as TLR3, which can detect viral RNA to trigger a type I IFN response by the TRIF signaling pathway. In addition, TLR7 and TLR8 detect viral RNA while TLR9 detects CpG containing viral DNA in the endosomes triggering the same response through the MyD88 signaling pathway (Kawai and Akira, 2006). In addition to TLRs, there are several cytosolic receptors including RIG-Ilike receptors that recognizes viral RNA, as well as cytosolic DNA sensors such as cyclic GMP-AMP synthase, AIM2-like proteins and DNA-dependent activator of IFN-regulatory factors that produces an IFN response (Goubau et al., 2013). It is likely that additional candidates are present as redundancy is built into viral sensing mechanisms in host cells (Nish and Medzhitov, 2011).

ABCF1, a member of the ATP Binding Cassette family expressed in diverse mammals and different tissue types, has been reported to have diverse functions including initiation of mRNA translation, dsDNA viral sensing, and polarization of immune cell phenotype (Richard et al., 1998; Tyzack et al., 2000; Paytubi et al., 2008, 2009; Lee et al., 2013; Arora et al., 2019). We have recently reported ABCF1 gene expression levels in human airway epithelium (Aguiar et al., 2019), but the function of this molecule remained unexplored. Herein we confirm ABCF1 gene and protein expression in situ and in vitro in primary human lung tissue and cell lines and explore its function in airway epithelial cells. Under basal conditions, reduced expression of ABCF1 did not lead to quantitative changes in cell viability or qualitative changes in cell morphology associated with cell death. Furthermore, ABCF1 reduction failed to significantly alter basal transcriptional activity in a human airway epithelial cell line, HBEC-6KT. Under VACV-70 challenge, a model of dsDNA viral exposure, ABCF1 was linked to CXCL10 secretion. Interestingly, despite the demonstrated activation of a viral gene signature by VACV-70, no global change in antiviral gene 
A

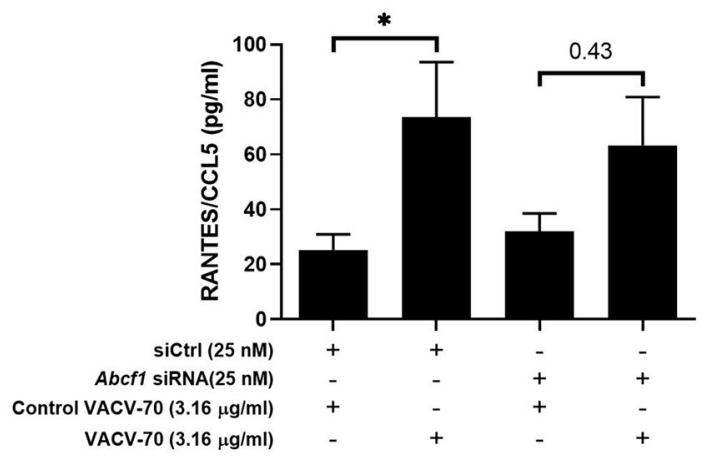

C

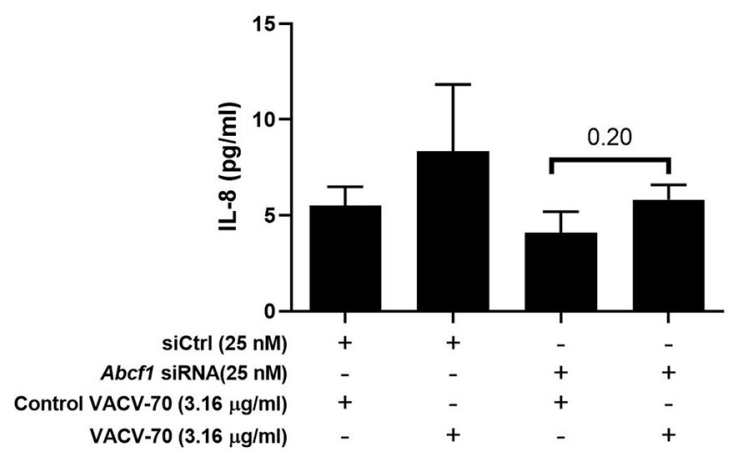

E

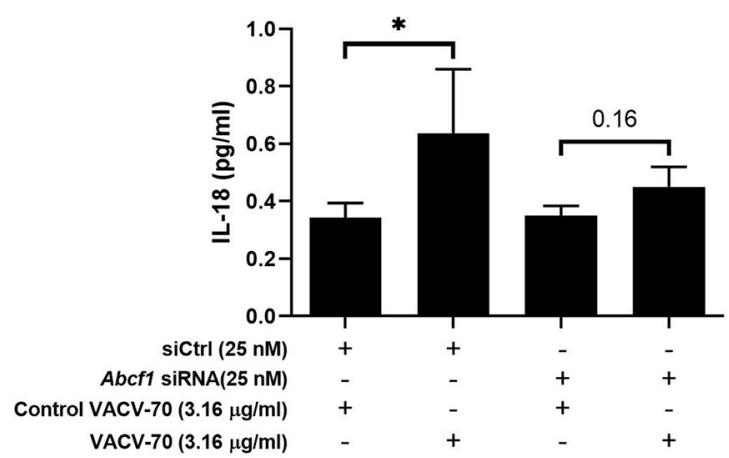

G

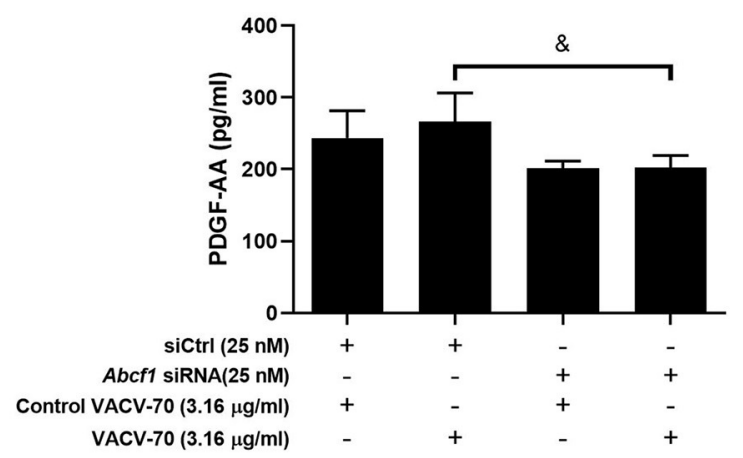

B

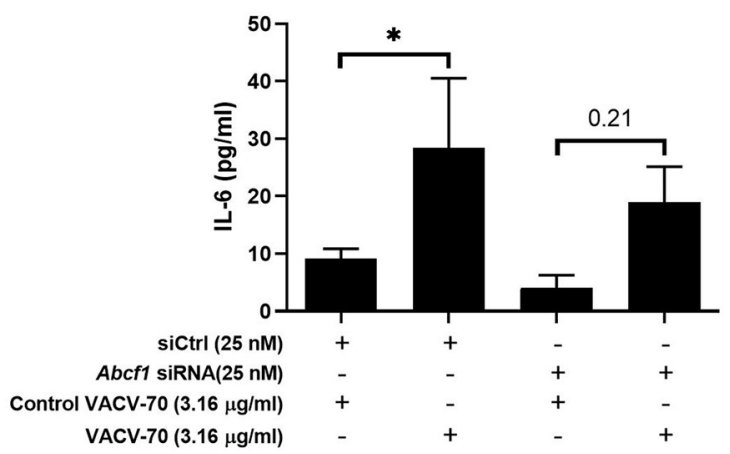

D

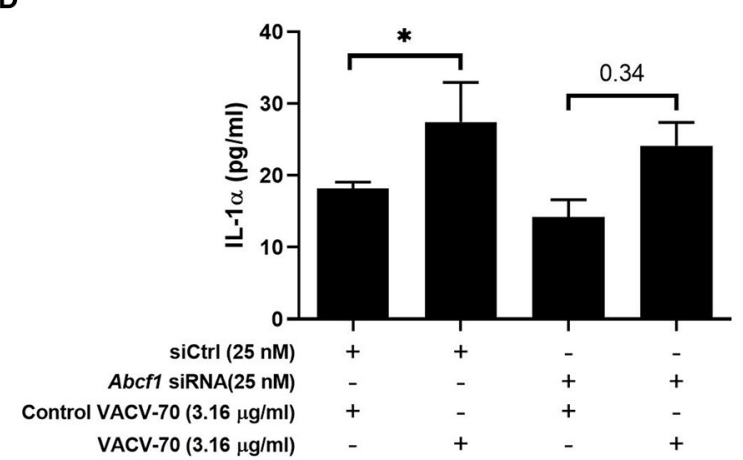

F

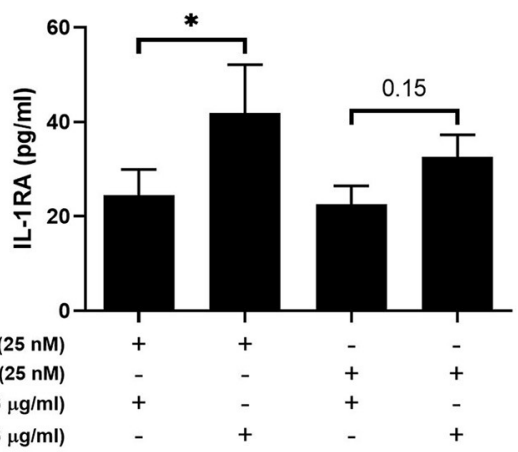

H

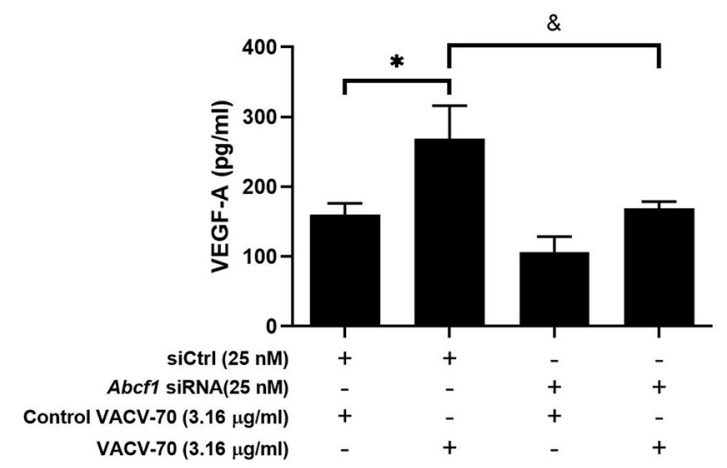

FIGURE 5 | Impact of ABCF1 reduction on VACV-70 stimulated cytokine production-Inflammatory cytokines and growth factors. VACV-70 (3.16 $\mu \mathrm{g} / \mathrm{mll})$-induced cytokine protein production for HBEC-6KT cells with ABCF1 siRNA treatment. (A) RANTES/CCL5, (B) IL-6, (C) IL-8, (D) IL-1a, (E) IL-18, (F) IL-1RA, (G) PDGF-AA, (H) VEGF-A. All studies $n=3$; ${ }^{*} p<0.05$ relative to siCtrl + ctrl VACV-70; $\&=p<0.05$ relative to siCtrl+VACV-70. 


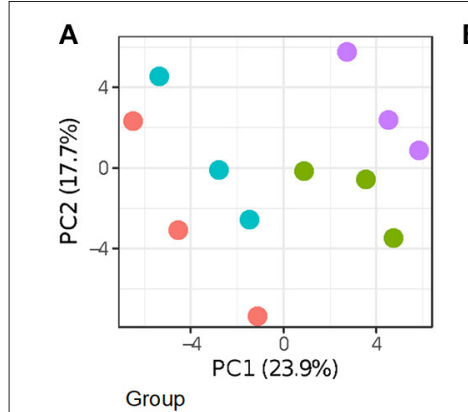

- siCtrl + Control VACV-70

- sictrl + VACV-70

- ABCF1 siRna + Control VACV-70

- ABCF1 siRNA + VACV-70

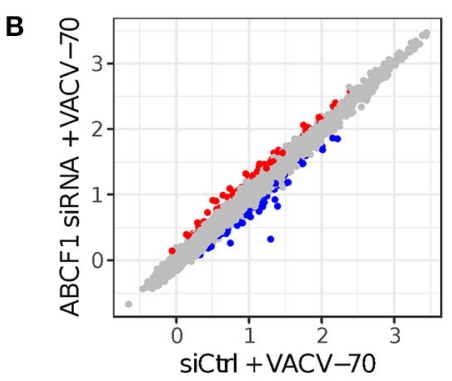

- NS

- Down

- Up
C

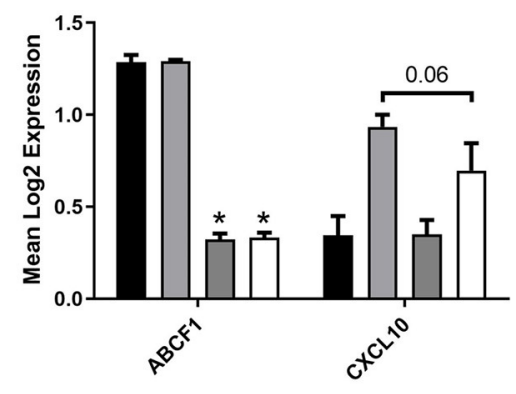

$$
\begin{aligned}
& \text { siCtrl + Control VACV-70 } \\
& \text { siCtrl + VACV-70 } \\
& \text { Abcf1 siRNA + Control VACV-70 }
\end{aligned}
$$$$
\square \text { Abcf1 siRNA + VACV-70 }
$$

D

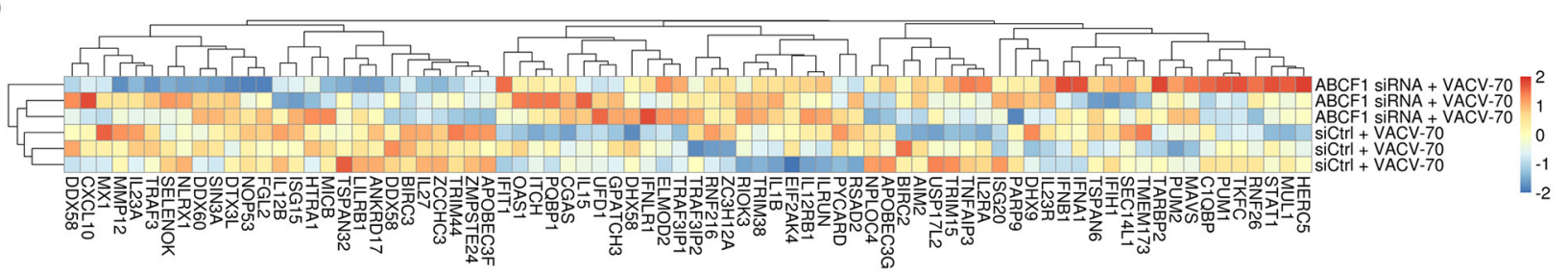

E

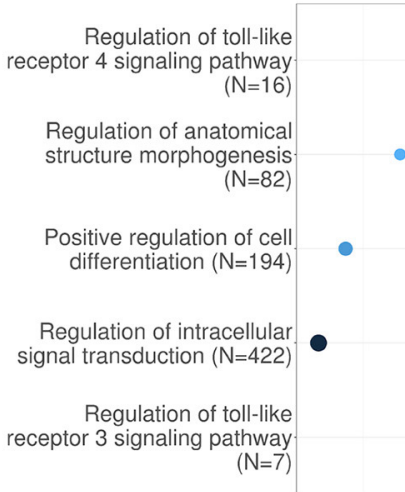

${ }^{100}$ Combined Score 200

G

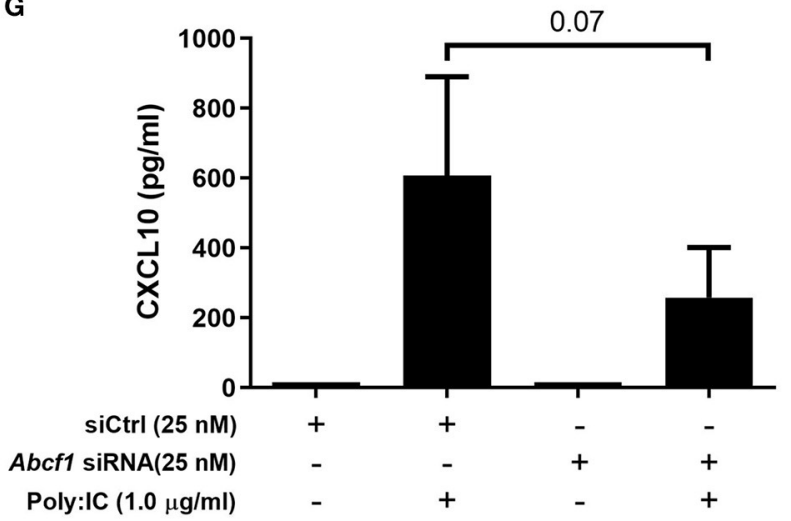

F

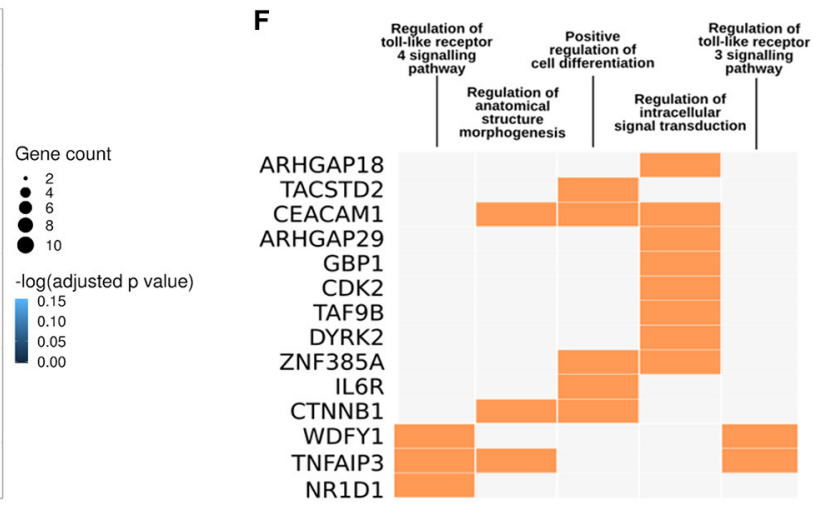

H

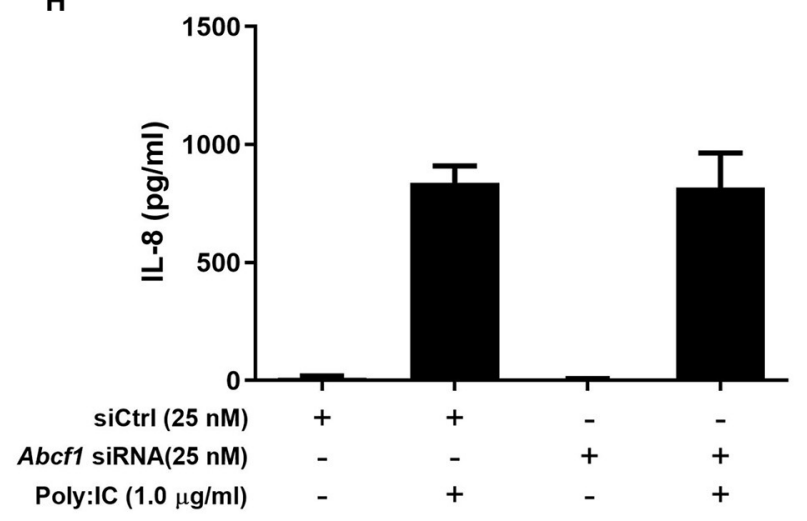

FIGURE 6 | Transcriptional interrogation of ABCF1 function during VACV-70 challenge in HBEC-6KT. (A) PCA plot of microarray gene expression profiles of HBEC-6KT cells following ABCF1 knockdown and VACV-70 treatment. Red circles (control siRNA and control VACV-70), green circles (control siRNA and VACV-70), 
FIGURE 6 | blue circles (ABCF1 siRNA and control VACV-70), purple circles (ABCF1 siRNA and VACV-70). (B) Log 2 expression data for transfection treatment with ABCF1 siRNA and VACV-70 compared to control siRNA and VACV-70. Significantly differently expressed genes are identified in blue (down-65 genes) and red (up-63 genes). (C) Confirmation of ABCF1 and CXCL10 attenuation with ABCF1 siRNA treatment presented as log 2 expression data. Black bars: control siRNA and control VACV-70, light gray bars: control siRNA and VACV-70, dark gray bars: ABCF1 siRNA and control VACV-70, white bars: ABCF1 siRNA and VACV-70. (D) Heat map of $\log _{2}$ expression data (scaled by gene) of genes associated with the "regulation of defense response to virus" GO term ( $n=68$ ) plus the selected known antiviral genes from Figure 3E $(n=11)$ for VACV-70 samples with and without ABCF1 siRNA. (E) Top 5 GO Biological Processes are ranked by increasing -log 10 adjusted $p$-value, with number (Count) of significantly differentially expressed genes between VACV-70 samples with and without ABCF1 siRNA, contributing to the total number of genes associated with the given pathway $(N)$ denoted by the size of circle. (F) Significantly differentially expressed genes between VACV-70 samples with and without ABCF1 were submitted to Enrichr for generation of a clustergram defining the gene contribution (Y-axis) to the functional enrichment of the top $5 \mathrm{GO}$ Biological Processes $(X$-axis), with orange squares denoting the association of a differentially expressed gene with a particular GO term. (G) Poly I:C (1.0 $\mu \mathrm{g} / \mathrm{ml})$-induced CXCL10 and $\mathbf{( H )}$ IL-8 secretion for HBEC-6KT cells with ABCF1 siRNA treatment. All studies $n=3 ;{ }^{*} p<0.05$.

expression patterns were observed with $A B C F 1$ reduction. In contrast, the gene pathways regulated by ABCF1 under VACV70 challenge were associated with TLR signaling and intracellular signal transduction. Furthermore, Poly I:C, a dsRNA analog and TLR3 ligand induced CXCL10 in an ABCF1 sensitive mechanism. Collectively, our data suggests that ABCF1 may regulate CXCL10 production downstream of dsDNA sensing mechanisms and TLR3 in human airway epithelial cells. It remains possible that $\mathrm{ABCF} 1$ can function to complement viral sensing mechanisms mediated by canonical dsRNA viral response machinery (e.g., RIG-I) as there are possible redundancies in viral sensing mechanisms in the cell (Nish and Medzhitov, 2011).

ABCF1 (originally called ABC50) was first identified in human synoviocytes at the mRNA level as a transcript regulated by TNF- $\alpha$ exposure (Richard et al., 1998). ABCF1 is unique in the mammalian $\mathrm{ABC}$ transporter family in that it contains the signature ATP binding LSGGQ amino acid motif and associated Walker A and B motifs for phosphate binding, but lacks a predicted transmembrane region (Richard et al., 1998; Loo et al., 2002; Higgins and Linton, 2004), which is supportive of a cytosolic localization and function. $A B C F 1$ transcript expression profiling has revealed near ubiquitous expression in human organs including lung, heart, brain, placenta, liver, skeletal muscle, kidney, pancreas, spleen, thymus, prostate, testis, ovary, small intestine, colon, peripheral blood leukocytes (Richard et al., 1998). The expression of ABCF1 has subsequently been identified in the human HeLa cells and embryonic kidney cells and other mammalian cells from rats, rabbits, hamsters, and mice (Tyzack et al., 2000; Paytubi et al., 2008, 2009; Wilcox et al., 2017; Arora et al., 2019). Highlighting the importance of ABCF1 in normal physiology and development, homozygous deletion of $\mathrm{ABCF} 1$ is embryonic lethal in either $\mathrm{C} 57 \mathrm{Bl} / 6$ mice or BALB/c mice (Wilcox et al., 2017). As our group recently identified gene expression of $A B C F 1$ in human airway epithelial cells (Aguiar et al., 2019), we set out to first confirm this at the protein level and then determine the function(s) of ABCF1 in human airway epithelial cells. We confirm that $A B C F 1$ gene expression is present in airway epithelial cells and expressed at levels relative to other known $\mathrm{ABC}$ transporters with function in this cell type, ABCC4 and ABCC7/CFTR (Riordan et al., 1989; Conner et al., 2013; Gold et al., 2016; Huff et al., 2017b). In situ hybridization using RNAscope ${ }^{\mathrm{TM}}$ technology demonstrated $A B C F 1$ transcripts present in the airway epithelial cells in human lung samples, which was consistent with positive immunohistochemical staining of protein in a serial section of the same samples using an antibody validated for specificity. Since the original discovery of ABCF1 was the result of an upregulated transcript resulting from TNF- $\alpha$ stimulation of synoviocytes, we examined if this mechanism was functional in human airway epithelial cells. In contrast to the reported data on synoviocytes, TNF- $\alpha$ stimulation failed to induce ABCF1 protein expression in HBEC-6KT, despite IL- 8 induction as a positive control. Interestingly, in a recent report profiling the role of $\mathrm{ABCF} 1$ in murine bone-marrow derived macrophages, TNF- $\alpha$ stimulation suppressed ABCF1 protein expression (Arora et al., 2019). These contrasting observations of ABCF1 expression in HBEC-6KT compared to synoviocytes and bone-marrow derived macrophages could be due to the difference in ontogeny and function of these cells. Collectively our results and those in the literature support gene and protein expression of ABCF1 in human airway epithelial cells, and that regulation of this protein is likely to be cell specific.

The first description of a potential function for ABCF1 in mammalian cells was derived from the experiments on human synoviocytes, suggesting a role in translation due to homology of molecular sequence with yeast proteins that performed this function (Richard et al., 1998; Tyzack et al., 2000). The embryonic lethality observed in mice for homozygous ABCF1 deletion and ubiquitous expression across multiple cell and tissue types (Wilcox et al., 2017), is consistent with ABCF1 playing a role in a fundamental biological process like protein translation. The observation that proliferating cells including synoviocytes stimulated with TNF- $\alpha$ and T cells stimulated with phorbol myristate acetate and ionomycin, elevate ABCF1 levels is further consistent with a role in translation (Richard et al., 1998; Tyzack et al., 2000). Subsequent to the discovery of ABCF1 gene expression and homology modeling, biochemical studies implicated the protein in interaction with eukaryotic initiation factor-2 (eIF2), a heterotrimeric protein consisting of $\alpha, \beta$, and $\gamma$ subunits, that is important for translation initiation (Tyzack et al., 2000). A distinguishing feature of ABCF1 relative to other $\mathrm{ABC}$ transporters is a $\mathrm{N}$-terminal domain that is able to interact with eIF $2 \alpha$ in a process that potentiates binding of methionyltRNA and initiation of translation (Paytubi et al., 2008). In addition to eIF2 $\alpha$ interactions, ABCF1 associates with ribosomes in a process potentiated by ATP binding to the nucleotide binding domains and inhibited by ADP (Tyzack et al., 2000), although the hydrolysis of ATP seems dispensable for ribosome 
interaction (Paytubi et al., 2008). To explore the potential function of $\mathrm{ABCF} 1$ as an initiator of translation in human airway epithelial cells, we undertook a siRNA approach to attenuate gene and protein expression followed by a global assessment of cell viability and transcriptomics. Surprisingly, under basal conditions, reduced expression of $\mathrm{ABCF} 1$ at the gene and protein level did not impact HBEC-6KT viability, morphology or transcriptional profile. Importantly, our outcome measurements were performed on HBEC-6KT that were sub-confluent and undergoing proliferation in serum-free media, an experimental condition where ABCF1 function in translation initiation would be relevant. A limitation of our design is that we measured global gene expression under the assumption that this would reflect any global changes in gene translation, an indirect approach which does not allow us to directly implicate ABCF1 expression levels to protein synthesis. Interestingly, our observations of minimal changes in HBEC-6KT may be consistent with cells of epithelial lineage, as near complete ABCF1 knockdown in HeLa cells was also only associated with a modest attenuation of total protein synthesis (Paytubi et al., 2009). Collectively, our results suggest that ABCF1 may function independent of protein translation functions in HBEC-6KT, as gene and protein attenuation results in no changes in cell viability or global transcriptional profile.

The original discovery that ABCF1 expression was regulated by TNF- $\alpha$ stimulation suggested a link to immune responses, although no differential expression patterns were observed for synoviocytes from healthy individuals or those with rheumatoid arthritis (Richard et al., 1998). Subsequently, ABCF1 has been implicated in immune responses via a cytosolic dsDNA viral sensing function using mouse embryonic fibroblasts (Lee et al., 2013). Using an integrative bioinformatic and molecular biology approach, a biotinylated ISD sequence was used as a bait and transfected into cells, followed by proteomic interrogation of identified candidates. The ISD bait method was validated by identifying known dsDNA sensors including HMGB1, HMGB2, and HMGB3, components of the AIM2 inflammasome, and the SET complex that plays a role in HIV-1 retroviral detection and infection (Yan et al., 2009). Within the pool of unknown dsDNA interacting candidates, $\mathrm{ABCF} 1$ was mechanistically linked to ISD induced-CXCL10 production using siRNA methods. The observed ISD induced-CXCL10 converged on IRF3 signaling, confirmed by showing reduced IRF3 phosphorylation following ISD treatment under conditions of ABCF1 silencing. In a separate study, ABCF1 has been implicated as a molecular switch downstream of TLR4 signaling in mouse bone-marrow derived macrophages that regulates MyD88 dependent pro-inflammatory and TRIF/TRAM dependent anti-inflammatory processes (Arora et al., 2019). Using in vitro an in vivo model systems, ABCF1 was implicated in polarizing pro-inflammatory macrophages to an anti-inflammatory/tolerant macrophage phenotype with direct involvement in shifting the systemic inflammatory response syndrome to a endotoxin tolerance phase in sepsis (Arora et al., 2019). The mechanism responsible for the ABCF1-mediated polarization of macrophages was identified to be a E2-ubiqutinconjugating enzyme function. In wild-type macrophages the TRIF-IFN- $\beta$ pathway is intact with attenuation of the MyD88 pathway, allowing IRF-3 phosphorylation, dimerization, and
IFN- $\beta$ expression. In contrast, heterozygosity for $\mathrm{ABCF} 1$ results in attenuation of the TRIF-IFN- $\beta$ pathway, with reduced IRF- 3 activation and IFN- $\beta$ production. Importantly, these two immunological studies converge on a relationship between ABCF1 and IRF3, which could involve direct or indirect interactions to facilitate downstream immune responses. Consistent with the potential role for ABCF1 as a dsDNA sensor, we explored immune and transcriptional responses downstream of VACV-70, a dsDNA viral mimic capable of activating STING, TBK1, and IRF3 independent of TLRs (Unterholzner et al., 2010). VACV-70 induced a dominant antiviral signature and pathway activation in HBEC-6KT, consistent with successful transfection and cytosolic sensing. Reduced expression of ABCF1 was associated with a reduction in CXCL10, an antiviral cytokine regulated by IRF3 activation, independent of any changes in cell viability or morphology. Transcriptomics revealed that although attenuation of CXCL10 was observed with ABCF1 siRNA, a global attenuation of an antiviral signature was not observed. Hypothesis-free GO analysis identified that the key pathways that were significantly impacted by ABCF1 siRNA treatment during VACV-70 challenge were related to TLR signaling. Interestingly, a key gene identified in our VACV-70 challenge and ABCF1 silencing studies is WDFY1, which links TLR3/4, TRIF, and IRF3 signaling (Hu et al., 2015). This finding suggested that ABCF1 could potentially be regulating both TLR4 and TLR3/TRIF/IRF3 signaling (Arora et al., 2019). We tested this hypothesis by using Poly I:C, a dsRNA viral mimic that activates TLR3 and IRF3 (Goubau et al., 2013). ABCF1 siRNA treatment attenuated Poly I:C-induced CXCL10 production, further demonstrating a link between ABCF1 and TRIF/IRF3, perhaps through regulation of WDYF1. While we have not yet demonstrated the link between ABCF1 and TLR4, a LPS challenge with ABCF1 siRNA treatment would effectively interrogate this. Our exploratory results suggest that $\mathrm{ABCF} 1$ is likely to play a complex role in innate immunity in response to cytosolic nucleic acids, with a potential interaction with TRIF/IRF3 for regulation of CXCL10.

Throughout our study we encountered several technical issues. The absence of pharmacological interventions that could antagonize $\mathrm{ABCF} 1$ function required us to pursue molecular approaches with siRNA. siRNA approaches were unable to completely attenuate ABCF1 at concentrations of $25 \mathrm{nM}$ for up to $48 \mathrm{~h}$. Longer durations of silencing were not possible as the human airway epithelial cell line used showed changes in morphology with vehicle control transfection reagent beyond $48 \mathrm{~h}$ of incubation. Our inability to completely attenuate ABCF1 levels was consistent with human embryonic kidney cells (Paytubi et al., 2009). Secondary to addressing ABCF1 expression levels, we sought to explore the functional consequences with the reported dsDNA viral mimic ISD as reported in the literature with mouse embryonic fibroblasts (Arora et al., 2019). During our concentration-response studies with ISD, the vehicle control condition resulted in elevations in our primary readout of CXCL10, which suggested an unexplained confounding factor. We therefore opted to use VACV-70 in place of ISD, which limits our ability to directly compare our results to those that have established ABCF1 as a dsDNA sensor with ISD (Arora et al., 2019). Importantly, reported findings from the HBEC-6KT 
cell line will require confirmation using primary human airway epithelial cells under submerged and/or air-liquid interface culture conditions to more accurately model in the in situ human respiratory mucosa.

In conclusion, we confirm that ABCF1 is expressed at the gene and protein level in situ and in vitro in human airway epithelial cells. In HBEC-6KT, ABCF1 has minimal functions for cell viability and transcriptional regulation under basal conditions but is important for mediating immune responses to cytosolic nucleic acids in pathways that involve TLR signaling and CXCL10 production. Our data form the foundation to pursue precisely how $\mathrm{ABCF} 1$ is regulated and where it functions in the network of cytosolic nucleic acid sensors and immune responses in human airway epithelial cells.

\section{DATA AVAILABILITY STATEMENT}

The datasets ANALYZED for this study can be found in the NCBI GEO accession GSE11906.

\section{ETHICS STATEMENT}

The studies involving human participants were reviewed and approved by Hamilton Integrated Research Ethics Board. The patients/participants provided their written informed consent to participate in this study.

\section{REFERENCES}

Aguiar, J. A., Tamminga, A., Lobb, B., Huff, R. D., Nguyen, J. P., Kim, Y., et al. (2019). The impact of cigarette smoke exposure, COPD, or asthma status on $\mathrm{ABC}$ transporter gene expression in human airway epithelial cells. Sci. Rep. 9:153. doi: 10.1038/s41598-018-36248-9

Altenburg, A. F., van Trierum, S. E., de Bruin, E., de Meulder, D., van de Sandt, C. E., van der Klis, F. R. M., et al. (2018). Effects of pre-existing orthopoxvirusspecific immunity on the performance of modified vaccinia virus ankara-based influenza vaccines. Sci. Rep. 8:6474. doi: 10.1038/s41598-018-24820-2

Arora, H., Wilcox, S. M., Johnson, L. A., Munro, L., Eyford, B. A., Pfeifer, C. G., et al. (2019). The ATP-binding cassette gene ABCF1 functions as an E2 ubiquitin-conjugating enzyme controlling macrophage polarization to dampen lethal septic shock. Immunity 50, 418-431.e416. doi: 10.1016/j.immuni.2019.01.014

Bailey, K. L., Romberger, D. J., Katafiasz, D. M., Heires, A. J., Sisson, J. H., Wyatt, T. A., et al. (2015). TLR2 and TLR4 expression and inflammatory cytokines are altered in the airway epithelium of those with alcohol use disorders. Alcohol. Clin. Exp. Res. 39, 1691-1697. doi: 10.1111/acer.12803

Bergelson, J. M., Cunningham, J. A., Droguett, G., Kurt-Jones, E. A., Krithivas, A., Hong, J. S., et al. (1997). Isolation of a common receptor for Coxsackie B viruses and adenoviruses 2 and 5. Science 275, 1320-1323. doi: 10.1126/science.275.5304.1320

Bewley, M. C., Springer, K., Zhang, Y. B., Freimuth, P., and Flanagan, J. M. (1999). Structural analysis of the mechanism of adenovirus binding to its human cellular receptor, CAR. Science 286, 1579-1583. doi: $10.1126 /$ science. 286.5444 .1579

Biggs, H. M., Lu, X., Dettinger, L., Sakthivel, S., Watson, J. T., and Boktor, S. W. (2018). Adenovirus-associated influenza-like illness among college students, Pennsylvania, USA. Emerg. Infect. Dis. 24, 2117-2119. doi: $10.3201 /$ eid2411.180488

Carlin, C. R. (2019). New insights to adenovirus-directed innate immunity in respiratory epithelial cells. Microorganisms 7:216. doi: 10.3390/microorganisms7080216

\section{AUTHOR CONTRIBUTIONS}

QC: designed, performed, and analyzed in vitro experiments, in vitro figure generation, drafting and editing of the manuscript. JA and BT: performed bioinformatics analysis, figure generation, and drafted the manuscript. NA: performed in vitro experiments, \& in vitro figure generation. NT: processed human tissue, performed in vitro experiments, \& in vitro figure generation. NM: responsible for patient consenting and human tissue acquisition. SR: responsible for histology and imaging. AA: responsible for human ethics protocols, human tissue acquisition, histology, and imaging. GC: responsible for supervision of the clinical research coordinator and human tissue acquisition. KA: responsible for supervision of the trainees, human tissue acquisition, study design, histology, and imaging. AD: responsible for oversight of the entire study \& supervision of the trainees and funding. $\mathrm{JH}$ : responsible for oversight of the entire study \& supervision of the trainees and funding, drafting and editing of the manuscript. All authors contributed to the article and approved the submitted version.

\section{FUNDING}

This work was supported by the Canadian Institutes of Health Research and the British Columbia Lung Association.

Chen, E. Y., Tan, C. M., Kou, Y., Duan, Q., Wang, Z., Meirelles, G. V., et al. (2013). Enrichr: interactive and collaborative HTML5 gene list enrichment analysis tool. BMC Bioinformatics 14:128. doi: 10.1186/1471-2105-14-128

Conner, G. E., Ivonnet, P., Gelin, M., Whitney, P., and Salathe, M. (2013). HO stimulates CFTR through an autocrine prostaglandin pathway using MRP4. Am. J. Respir. Cell Mol. Biol. 49, 672-679. doi: 10.1165/rcmb.2013-0 $1560 \mathrm{C}$

Dai, M., Wang, P., Boyd, A. D., Kostov, G., Athey, B., Jones, E. G., et al. (2005). Evolving gene/transcript definitions significantly alter the interpretation of genechip data. Nucleic Acids Res. 33:e175. doi: 10.1093/nar/gni179

de Vries, R. D., Altenburg, A. F., Nieuwkoop, N. J., de Bruin, E., van Trierum, S. E., Pronk, M. R., et al. (2018). Induction of cross-clade antibody and Tcell responses by a modified vaccinia virus ankara-based influenza A(H5N1) vaccine in a randomized phase 1/2a clinical trial. J. Infect. Dis. 218, 614-623. doi: 10.1093/infdis/jiy214

GBD 2017 Causes of Death Collaborators (2018). Global, regional, and national age-sex-specific mortality for 282 causes of death in 195 countries and territories, 1980-2017: a systematic analysis for the global burden of disease study 2017. Lancet 392, 1736-1788. doi: 10.1016/S0140-6736(18)3 2203-7

Gold, M. J., Hiebert, P. R., Park, H. Y., Stefanowicz, D., Le A, Starkey, M. R., Deane, A., et al. (2016). Mucosal production of uric acid by airway epithelial cells contributes to particulate matter-induced allergic sensitization. Mucosal Immunol. 9, 809-820. doi: 10.1038/mi.2015.104

Goubau, D., Deddouche, S., and Reise Sousa, C. (2013). Cytosolic sensing of viruses. Immunity 38, 855-869. doi: 10.1016/j.immuni.2013.05.007

Higgins, C. F., and Linton, K. J. (2004). The ATP switch model for ABC transporters. Nat. Struct. Mol. Biol. 11, 918-926. doi: 10.1038/nsmb836

Hirota, J. A., Gold, M. J., Hiebert, P. R., Parkinson, L. G., Wee, T., Smith, D., et al. (2015a). The nucleotide-binding domain, leucine-rich repeat protein 3 inflammasome/IL-1 receptor I axis mediates innate, but not adaptive, immune responses after exposure to particulate matter under 10 mum. Am. J. Respir. Cell Mol. Biol. 52, 96-105. doi: 10.1165/rcmb.2014-0 $158 \mathrm{OC}$ 
Hirota, J. A., Hirota, S. A., Warner, S. M., Stefanowicz, D., Shaheen, F., Beck, P. L., et al. (2012). The airway epithelium nucleotide-binding domain and leucine-rich repeat protein 3 inflammasome is activated by urban particulate matter. J. Allergy Clin. Immunol. 129, 1116-1125. doi: 10.1016/j.jaci.2011. 11.033

Hirota, J. A., and Knight, D. A. (2013). Human airway epithelial cell innate immunity: relevance to asthma. Curr. Opin. Immunol. 24, 740-746. doi: 10.1016/j.coi.2012.08.012

Hirota, J. A., Marchant, D. J., Singhera, G. K., Moheimani, F., Dorscheid, D. R., Carlsten, C., et al. (2015b). Urban particulate matter increases human airway epithelial cell IL-1beta secretion following scratch wounding and H1N1 influenza A exposure in vitro. Exp. Lung Res. 41, 353-362. doi: 10.3109/01902148.2015.1040528

Hodinka, R. L. (2016). Respiratory RNA viruses. Microbiol Spectr. 4, 233-271 doi: 10.1128/microbiolspec.DMIH2-0028-2016

Hu, Y. H., Zhang, Y., Jiang, L. Q., Wang, S., Lei, C. Q., Sun, M. S., et al. (2015). WDFY1 mediates TLR3/4 signaling by recruiting TRIF. EMBO Rep. 16, 447-455. doi: 10.15252/embr.201439637

Huber, W., Carey, V. J., Gentleman, R., Anders, S., Carlson, M., Carvalho, B. S., et al. (2015). Orchestrating high-throughput genomic analysis with bioconductor. Nat. Methods 12, 115-121. doi: 10.1038/nmeth.3252

Huff, R. D., Hsu, A. C., Nichol, K. S., Jones, B., Knight, D. A., Wark, P. A. B., et al. (2017a). Regulation of xanthine dehydrogensase gene expression and uric acid production in human airway epithelial cells. PLOS ONE. 12:e184260. doi: 10.1371/journal.pone.0184260

Huff, R. D., Rider, C. F., Yan, D., Newton, R., Giembycz, M. A., Carlsten, C., et al. (2017b). Inhibition of ABCC4 potentiates combination beta agonist and glucocorticoid responses in human airway epithelial cells. J. Allergy Clin. Immunol. 141, 1127-1130.e5. doi: 10.1016/j.jaci.2017.10.011

Ishikawa, H., Ma, Z., and Barber, G. N. (2009). STING regulates intracellular DNAmediated, type I interferon-dependent innate immunity. Nature 461, 788-792. doi: $10.1038 /$ nature 08476

Jartti, T., and Gern, J. E. (2017). Role of viral infections in the development and exacerbation of asthma in children. J. Allergy Clin. Immunol. 140, 895-906. doi: 10.1016/j.jaci.2017.08.003

Kawai, T., and Akira, S. (2006). Innate immune recognition of viral infection. Nat. Immunol. 7, 131-137. doi: 10.1038/ni1303

Kokturk, N., Bozdayi, G., Yilmaz, S., Dogan, B., Gulbahar, O., Rota, S., et al. (2015). Detection of adenovirus and respiratory syncytial virus in patients with chronic obstructive pulmonary disease: exacerbation versus stable condition. Mol. Med. Rep. 12, 3039-3046. doi: 10.3892/mmr.2015.3681

Kuleshov, M. V., Jones, M. R., Rouillard, A. D., Fernandez, N. F., Duan, Q., Wang, Z., et al. (2016). Enrichr: a comprehensive gene set enrichment analysis web server 2016 update. Nucleic Acids Res. 44, W90-97. doi: 10.1093/nar/g kw377

Le Boeuf, F., Diallo, J. S., McCart, J. A., Thorne, S., Falls, T., Stanford, M., et al. (2010). Synergistic interaction between oncolytic viruses augments tumor killing. Mol. Ther. 18, 888-895. doi: 10.1038/mt.2010.44

Lee, M. N., Roy, M., Ong, S. E., Mertins, P., Villani, A. C., Li, W., et al. (2013). Identification of regulators of the innate immune response to cytosolic DNA and retroviral infection by an integrative approach. Nat. Immunol. 14, 179-185. doi: 10.1038/ni.2509

Linden, D., Guo-Parke, H., Coyle, P. V., Fairley, D., McAuley, D. F., Taggart, C. C., et al. (2019). Respiratory viral infection: a potential "missing link" in the pathogenesis of COPD. Eur. Respir. Rev. 28:180063. doi: 10.1183/16000617.0063-2018

Loo, T. W., Bartlett, M. C., and Clarke, D. M. (2002). The "LSGGQ" motif in each nucleotide-binding domain of human P-glycoprotein is adjacent to the opposing walker A sequence. J. Biol. Chem. 277, 41303-41306. doi: 10.1074/jbc.C200484200

Nish, S., and Medzhitov, R. (2011). Host defense pathways: role of redundancy and compensation in infectious disease phenotypes. Immunity 34, 629-636. doi: 10.1016/j.immuni.2011.05.009

Parker, D., and Prince, A. (2011). Innate immunity in the respiratory epithelium. Am. J. Respir. Cell Mol. Biol. 45, 189-201. doi: 10.1165/rcmb.2011-0011RT

Paytubi, S., Morrice, N. A., Boudeau, J., and Proud, C. G. (2008). The Nterminal region of $\mathrm{ABC} 50$ interacts with eukaryotic initiation factor eIF2 and is a target for regulatory phosphorylation by CK2. Biochem. J. 409, 223-231. doi: 10.1042/BJ20070811

Paytubi, S., Wang, X., Lam, Y. W., Izquierdo, L., Hunter, M. J., Jan, E., et al. (2009). ABC50 promotes translation initiation in mammalian cells. J. Biol. Chem. 284, 24061-24073. doi: 10.1074/jbc.M109.031625

Piccolo, S. R., Sun, Y., Campbell, J. D., Lenburg, M. E., Bild, A. H., and Johnson, W. E. (2012). A single-sample microarray normalization method to facilitate personalized-medicine workflows. Genomics 100, 337-344. doi: 10.1016/j.ygeno.2012.08.003

Proud, D., and Leigh, R. (2012). Epithelial cells and airway diseases. Immunol. Rev. 242, 186-204. doi: 10.1111/j.1600-065X.2011.01033.x

Raman, T., O’Connor, T. P., Hackett, N. R., Wang, W., Harvey, B. G., Attiyeh, M. A., et al. (2009). Quality control in microarray assessment of gene expression in human airway epithelium. BMC Genomics 10:493. doi: 10.1186/1471-2164-10-493

Ramirez, R. D., Sheridan, S., Girard, L., Sato, M., Kim, Y., Pollack, J., et al. (2004). Immortalization of human bronchial epithelial cells in the absence of viral oncoproteins. Cancer Res. 64, 9027-9034. doi: 10.1158/0008-5472.CAN-04-3703

Rich, D. P., Anderson, M. P., Gregory, R. J., Cheng, S. H., Paul, S., Jefferson, D. M., et al. (1990). Expression of cystic fibrosis transmembrane conductance regulator corrects defective chloride channel regulation in cystic fibrosis airway epithelial cells. Nature 347, 358-363. doi: 10.1038/347358a0

Richard, M., Drouin, R., and Beaulieu, A. D. (1998). ABC50, a novel human ATP-binding cassette protein found in tumor necrosis factor-alpha-stimulated synoviocytes. Genomics 53, 137-145. doi: 10.1006/geno.1998.5480

Riordan, J. R., Rommens, J. M., Kerem, B., Alon, N., Rozmahel, R., Grzelczak, Z., et al. (1989). Identification of the cystic fibrosis gene: cloning and characterization of complementary DNA. Science 245, 1066-1073. doi: 10.1126/science. 2475911

Ritchie, A. I., Farne, H. A., Singanayagam, A., Jackson, D. J., Mallia, P., and Johnston, S. L. (2015). Pathogenesis of viral infection in exacerbations of airway disease. Ann. Am. Thorac. Soc. 12 (Suppl, 2), S115-132. doi: 10.1513/AnnalsATS.201503-151AW

Ritchie, M. E., Phipson, B., Wu, D., Hu, Y., Law, C. W., Shi, W., et al. (2015). Limma powers differential expression analyses for RNA-sequencing and microarray studies. Nucleic Acids Res. 43:e47. doi: 10.1093/nar/g kv007

Shulenin, S., Nogee, L. M., Annilo, T., Wert, S. E., Whitsett, J. A., and Dean, M. (2004). ABCA3 gene mutations in newborns with fatal surfactant deficiency. N. Engl. J. Med. 350, 1296-1303. doi: 10.1056/NEJMoa0 32178

Singanayagam, A., Glanville, N., Girkin, J. L., Ching, Y. M., Marcellini, A., Porter, J. D., et al. (2018). Corticosteroid suppression of antiviral immunity increases bacterial loads and mucus production in COPD exacerbations. Nat. Commun. 9:2229. doi: 10.1038/s41467-018-04574-1

Stetson, D. B., and Medzhitov, R. (2006). Recognition of cytosolic DNA activates an IRF3-dependent innate immune response. Immunity 24, 93-103. doi: 10.1016/j.immuni.2005.12.003

Tyzack, J. K., Wang, X., Belsham, G. J., and Proud, C. G. (2000). ABC50 interacts with eukaryotic initiation factor 2 and associates with the ribosome in an ATP-dependent manner. J. Biol. Chem. 275, 34131-34139. doi: 10.1074/jbc.M002868200

Unterholzner, L., Keating, S. E., Baran, M., Horan, K. A., Jensen, S. B., Sharma, S., et al. (2010). IFI16 is an innate immune sensor for intracellular DNA. Nat. Immunol. 11, 997-1004. doi: 10.1038/ni.1932

Vasiliou, V., Vasiliou, K., and Nebert, D. W. (2009). Human ATPbinding cassette (ABC) transporter family. Hum. Genomics 3, 281-290. doi: 10.1186/1479-7364-3-3-281

Vermeer, P. D., McHugh, J., Rokhlina, T., Vermeer, D. W., Zabner, J., and Welsh, M. J. (2007). Vaccinia virus entry, exit, and interaction with differentiated human airway epithelia. J. Virol. 81, 9891-9899. doi: 10.1128/JVI.00 601-07

Viniol, C., and Vogelmeier, C. F. (2018). Exacerbations of COPD. Eur. Respir. Rev. 27:170103. doi: 10.1183/16000617.0103-2017

Wilcox, S. M., Arora, H., Munro, L., Xin, J., Fenninger, F., Johnson, L. A., et al. (2017). The role of the innate immune response regulatory gene ABCF1 
in mammalian embryogenesis and development. PLOS ONE 12:e0175918. doi: 10.1371/journal.pone.0175918

Yan, N., Cherepanov, P., Daigle, J. E., Engelman, A., and Lieberman, J. (2009). The SET complex acts as a barrier to autointegration of HIV-1. PLoS Pathog 5:e1000327. doi: 10.1371/journal.ppat.10 00327

Zhu, J., Message, S. D., Mallia, P., Kebadze, T., Contoli, M., Ward, C. K., et al. (2019). Bronchial mucosal IFN-alpha/beta and pattern recognition receptor expression in patients with experimental rhinovirus-induced asthma exacerbations. J. Allergy Clin. Immunol. 143, 114-125.e114. doi: 10.1016/j.jaci.2018.04.003
Conflict of Interest: The authors declare that the research was conducted in the absence of any commercial or financial relationships that could be construed as a potential conflict of interest.

Copyright ( 2020 Cao, Aguiar, Tremblay, Abbas, Tiessen, Revill, Makhdami, Ayoub, Cox, Ask, Doxey and Hirota. This is an open-access article distributed under the terms of the Creative Commons Attribution License (CC BY). The use, distribution or reproduction in other forums is permitted, provided the original author(s) and the copyright owner(s) are credited and that the original publication in this journal is cited, in accordance with accepted academic practice. No use, distribution or reproduction is permitted which does not comply with these terms. 